\title{
Layer Response Theory: Energetics of layered materials from semi-analytic high-level theory
}

\author{
John F Dobson, ${ }^{1,2}$ Tim Gould, ${ }^{1}$ and Sébastien Lebègue ${ }^{3}$ \\ ${ }^{1}$ Queensland Micro and Nano Technology Centre, \\ Griffith University, Nathan, Queensland 4111, Australia \\ ${ }^{2}$ Université de Lorraine, Vandoeuvre-lès-Nancy, F-54506, France \\ ${ }^{3}$ Laboratoire de Cristallographie, Résonance Magnétique et Modélisations (CRM2, \\ UMR CNRS 7036) Institut Jean Barriol, Université de Lorraine BP 239, \\ Boulevard des Aiguillettes 54506 Vandoeuvre-lès-Nancy,France
}

\begin{abstract}
We present a readily computable semi-analytic "Layer response theory" (LRT) for analysis of cohesive energetics involving two-dimensional layers such as BN or graphene. The theory approximates the Random Phase Approximation (RPA) correlation energy. Its RPA character ensures that the energy has the correct van der Waals asymptotics for well-separated layers, in contrast to simple pairwise atom-atom theories, which fail qualitatively for layers with zero electronic energy gap. At the same time our theory is much less computationally intensive than the full RPA energy. It also gives accurate correlation energies near to the binding minimum, in contrast to Lifshitz-type theory. We apply our LRT theory successfully to graphite and to BN, and to a graphene-BN heterostructure.
\end{abstract}

PACS numbers: 73.21.Ac,73.22.Pr,71.15.-m,71.15.Nc

\section{INTRODUCTION}

The interest in two dimensional compounds such as graphene ${ }^{1}$, hexagonal boron nitride ${ }^{2,3}$, transition metal dichalcogenides $^{2-4}$, and other promising compounds has grown exponentially over the last few years ${ }^{5-8}$. In particular, there has been much recent interest in their cohesive properties and also in layered, heterostructured solids composed of loosely-bound stacks of such 2 D layers ${ }^{9}$. Interesting properties include for example the binding energetics of atoms or molecules on such systems ${ }^{10-12}$, and the energetics of binding ${ }^{13}$ of the layer systems themselves. Possible applications include gas separation and storage technologies, ${ }^{14}$ and nano-electro-mechanical devices ${ }^{15-17}$.

The binding energetics of these layer systems typically show a significant contribution from van der Waals (vdW) forces, which renders their description by semilocal density functionals inaccurate. Much recent effort has therefore been put into simple vdW corrections, most typically involving a pairwise atom-based sum of vdW-type $R^{-6}$ atomatom interactions ${ }^{18-23}$. Some more sophisticated theories also can be expressed via pairwise summation ${ }^{24}$. While such pairwise and triples approaches are computationally efficient and often have good success with the inner, close-contact parts of the binding energy curves, it is known that they give an incorrect description of the far-distant energetics, especially for layers that are highly polarizable, metallic or semi-metallic ${ }^{25-29}$.

A relatively successful approach is the full numerical (direct) Random Phase Approximation ((d)RPA) correlation energy method ${ }^{30-34}$, henceforth denoted simply "RPA". This method and its extensions ${ }^{35},{ }^{36}$ have been found to give the best and most consistent treatment to date of energy differences in layered systems at all layer spacings provided electromagnetic retardation and higher many-body effects ${ }^{37}$ are are unimportant. ${ }^{38,39}$. In particular, the RPA approach correctly describes the very long-wavelength charge fluctuations not captured in pairwise theories, but responsible for anomalous long-ranged van der Waals interactions ${ }^{25}$. These differences from the pairwise predictions are manifested in both the dependence of the energy on layer spacing $D^{25,40}$ and, for finite systems, on the number of atoms $N^{27,29}$. Furthermore, the RPA (without extensions) has been shown ${ }^{33}$ to give simultaneously a good description of the layer spacing, the breathing elastic constant $C_{33}$ and the layer binding energy of graphite, a task that proved elusive with other methods. The RPA correlation approach, however, comes at a high computational cost that makes the analysis of complex systems difficult or impossible.

\section{LAYER RESPONSE THEORY}

In the present work we aim to produce a simplified, computationally efficient theory of the outer parts of the RPA correlation energy curves involving nano-thin layers. We then introduce a simple extension allowing analysis right down to intimate contact between layers.

For macrospically thick systems (slabs) there is already a very good theory of this type for the outer parts of the energy curve, namely the Lifshitz approach ${ }^{41-43}$ which describes non-contacting systems but, unlike the pure RPA, is not applicable to intimately contacting cases. The Lifshitz approach, rooted in quantum electrodynamics, has been 
further elaborated recently by a number of authors, e.g. refs 42,44-47. It uses the long-wavelength dielectric properties of the infinite solid as input (i.e. these are not predicted by Lifshitz theory, and must be obtained from experiment or independent calculations). The electromagnetically non-retarded limit of Lifshitz theory can alternatively be obtained by using an RPA-style correlation energy treatment ${ }^{48}$ to predict the van der Waals interaction between two semiinfinite solids. The RPA correlation energy approach, and our simplification of it described here, have a distinct advantage over traditional Lifshitz theory, however, in that they both remain accurate at small inter-layer separations right down to the binding distance.

The present theory is nevertheless somewhat similar in spirit to Lifshitz theory, in that it evaluates semi-analytically the outer parts of the energy-versus distance curves, using the long-wavength dielectric properties of a single layer as input. An important aspect of our approach, however, is that, rather than attempting to treat a monolayer as a very thin 3D macroscopic dielectric as one might do in Lifshitz theory, we carefully evaluate the realistic dielectric properties of a single 2D layer at the RPA level to the lowest three orders in a wavenumber expansion. We obtain this data using RPA-level calculation of the long-wavelength macroscopic dielectric function of a slightly stretched layered solid made from the individual layers under study. Such a calculation is now routinely available with great computational efficiency in modern ab-initio $3 \mathrm{D}$ codes (in our case $\mathrm{VASP}^{49,50}$ ). In particular, we do not require expensive calculations with large vacuum spaces between the layers and consequently large unit cells, because we show below how to deconvolute the Coulomb interactions between the layers analytically (see also the recent work of Nazarov $^{51}$ and of Andersen et al. $\left.{ }^{52}\right)$.

We take the long-wavelength $O\left(Q^{0}\right)$ layer polarizability data so obtained and augment it via analytic reasoning and a fitted parameter to obtain the $O\left(Q^{1}\right)$ and $O\left(Q^{2}\right)$ contributions to the polarizability respectively, for each species of monolayer. The $O\left(Q^{2}\right)$ term puts our theory well beyond the usual Lifshitz theory, which assumes strictly local dielectric properties. It allows us to obtain the correlation energy semi-analytically for systems approaching the contact configuration. We term our approach the "Layer Response Theory" of interactions in our target systems. We first introduce the electronic response functions of a single layer to external longitudinal fields, in a particularly convenient form, namely reflection and (differential) transmission coefficients $R(Q, \omega)$ and $T(Q, \omega)$, and then relate these to screened layer polarizabilities $\alpha^{2 D, s c r}$. The reflection/transmission (more generally, scattering) approach is in fact the popular modern way to implement Lifshitz theory ${ }^{44}$.

\section{REFLECTION AND TRANSMISSION OF ELECTROSTATIC FIELDS BY AN ISOLATED LAYER}

We define a "layer", labelled "I", of a material that has a periodic structure in the $x$ and $y$ spatial directions and is confined to a finite region $\mathcal{R}_{I}$ of the $z$ axis, containing a reference point $Z_{I} \in \mathcal{R}_{I}$. (See Fig 1$)$. By "confined" we mean that the groundstate electronic number density $n_{0 I}(\vec{r})$ and any linear time-dependent density perturbations $\delta n_{I}$ to it are assumed to vanish outside $\mathcal{R}_{I}$. In a perturbed situation, we can decompose the time-dependent density perturbations $\delta n_{I}$ into components varying as $\exp (i(\vec{Q}+\vec{G}) \cdot \vec{r}) \exp (u t) \delta n_{I}(\vec{Q}+\vec{G}, z, i u) \cdot$ Here $\vec{G}=G_{x} \vec{i}+G_{y} \vec{j}$ is a reciprocal lattice vector of the $2 \mathrm{D}$ periodic lattice, and $\vec{Q}=q_{x} \vec{i}+q_{y} \vec{j}$ is a $2 \mathrm{D}$ wavenumber lying inside the corresponding 2D Brillouin zone. Such a density perturbation will set up potentials $\delta W$ outside the layer given by

$$
\delta W_{\text {due to } I}(x, y, z)= \begin{cases}m_{I}^{+} \exp (i(\vec{Q}+\vec{G}) \cdot \vec{r}) \frac{2 \pi e^{2}}{|\vec{Q}+\vec{G}|} e^{-|\vec{Q}+\vec{G}|\left(z-Z_{I}\right)}, & z \text { to right of } \mathcal{R}_{I} \\ m_{I}^{-} \exp (i(\vec{Q}+\vec{G}) \cdot \vec{r}) \frac{2 \pi e^{2}}{|\vec{Q}+\vec{G}|} e^{+|\vec{Q}+\vec{G}|\left(z-Z_{I}\right)}, \quad z \text { to left of } \mathcal{R}_{I}\end{cases}
$$

Here the two moments

$$
\begin{aligned}
& m_{I}^{+}(\vec{Q}, \vec{G}, i u)=\int_{\mathcal{R}_{I}} e^{|\vec{Q}+\vec{G}|\left(z-Z_{I}\right)} \delta n_{I}(\vec{Q}, \vec{G}, z, i u) d z \\
& m_{I}^{-}(\vec{Q}, \vec{G}, i u)=\int_{\mathcal{R}_{I}} e^{-|\vec{Q}+\vec{G}|\left(z-Z_{I}\right)} \delta n_{I}(\vec{Q}, \vec{G}, z, i u) d z
\end{aligned}
$$

completely specify (via Eq (1)) the effects of layer $I$ on non-overlapping objects such as other layers or physisorbed atoms. From here onwards we assume initially that the matter in layer $I$ is sufficiently well separated from the edges of its region $\mathcal{R}_{I}$ that the rapidly (exponentially) decaying $\vec{G} \neq \overrightarrow{0}$ components in Eq (1) are negligible outside $\mathcal{R}_{I}$. See Fig 1. (We do modify this assumption for our final theory, however) Thus initially we only need the $\vec{G}=\overrightarrow{0}$ moments, which we shall denote

$$
m_{I}^{ \pm} \equiv m_{I}^{ \pm}(\vec{Q}, \vec{G}=\overrightarrow{0}, i u)=\int_{\mathcal{R}_{I}} e^{ \pm|\vec{Q}|\left(z-Z_{I}\right)} \delta n_{I}(\vec{Q}, \vec{G}=\overrightarrow{0}, z, i u) d z
$$


The potential $V_{I}(z) \exp (i \vec{Q} \cdot \vec{r}) \exp (u t)$ due to sources outside layer $I$ and acting on the matter inside layer $I$, is assumed to lack the rapidly (exponentially) damped $G \neq 0$ components because of the isolation of the matter deep enough inside layer $I$. This potential has no sources inside layer $I$ and hence obeys Poisson's equation $\nabla^{2} V_{I}=$ $\left(-Q^{2}+d^{2} / d z^{2}\right) V_{I}=0$ for $z \in \mathcal{R}_{I}$. Thus it can be completely specified by two amplitudes $V_{I}^{ \pm}$:

$$
V^{e x t}(z)=V_{I}^{+} e^{|\vec{Q}| z}+V_{I}^{-} e^{-|\vec{Q}| z}, \quad z \in \mathcal{R}_{I}
$$

Suppose that a small external field impinges on layer $I$ from the right, so that $V_{I}^{-}=0$ in (3). This potential gives rise to a density perturbation inside layer $I$

$$
\delta n_{I}(z)=\int_{\mathcal{R}_{I}} d z^{\prime} \chi_{I}\left(Q, \vec{G}=\vec{G}^{\prime}=\overrightarrow{0}, z, z^{\prime}, i u\right) V_{I}^{+} e^{|\vec{Q}|\left(z^{\prime}-Z_{I}\right)}
$$

where the finite-frequency density-density response $\chi_{I}$ of the layer has to include all Coulomb screening and local field effects inside layer $I$.

The density (4) in turn creates a reflected field to the right of $\mathcal{R}_{I}$ of form

$$
V^{r e f l, r}=R_{I}^{r i g h t} V_{I}^{+} \exp (i \vec{Q} \cdot \vec{r}) \exp \left(-Q\left(z-Z_{I}\right)\right), \quad z \text { to right of } \mathcal{R}_{I}
$$

where, from (1) and (4), the dimensionless "right hand reflection coefficient" $R_{I}^{\text {right }}$ is

$$
R_{I}^{r i g h t}=\frac{2 \pi e^{2}}{Q} \int_{\mathcal{R}_{I}} d z d z^{\prime} e^{Q\left(z+z^{\prime}-2 Z_{I}\right)} \chi_{I}\left(\vec{Q}, z, z^{\prime}, i u\right)
$$

Similarly, still with a potential $V_{I}^{+}$impinging from the right, layer $I$ also creates an additional "transmitted" potential to its left

$$
V^{\text {tr, right }}=T_{I}^{\text {right }} V_{I}^{+} \exp (i \vec{Q} \cdot \vec{r}) \exp \left(Q\left(z-Z_{I}\right)\right), \quad z \text { to left of } \mathcal{R}_{I}
$$

where the dimensionless "right (differential) transmission coefficient" is

$$
T_{I}^{r i g h t}=\frac{2 \pi e^{2}}{Q} \int_{\mathcal{R}_{I}} d z d z^{\prime} e^{Q\left(-z+z^{\prime}\right)} \chi_{I}\left(\vec{Q}, z, z^{\prime} i u\right)
$$

Similarly for fields impinging on layer $I$ from the left we have "left" reflection and transmission coefficients

$$
\begin{gathered}
R_{I}^{\text {left }}=\frac{2 \pi e^{2}}{Q} \int_{\mathcal{R}_{I}} d z d z^{\prime} e^{-Q\left(z+z^{\prime}-2 Z_{I}\right)} \chi_{I}\left(\vec{Q}, z, z^{\prime}, i u\right) \\
T_{I}^{\text {left }}=\frac{2 \pi e^{2}}{Q} \int_{\mathcal{R}_{I}} d z d z^{\prime} e^{Q\left(z-z^{\prime}\right)} \chi_{I}\left(\vec{Q}, z, z^{\prime}, i u\right)
\end{gathered}
$$

For a layer with inversion symmetry in the $z$ direction, the distinction between fields impinging from left or right disappears, and we just have a reflection coefficient $R_{I}$ and and a transmission coefficient $T_{I}$ given by (6) and (8).

It is useful to re-express these reflection and transmission coefficients in terms of an interacting or screened polarizability $a_{i j}^{s c r}$ giving the change in polarization $p_{i}$ (dipole moment per unit volume) per unit applied external electric field $E_{j}^{e x t}$. By contrast the above quantity $\chi$ gives the response of the number density $\delta n$ to a unit external electronic potential energy perturbation $\delta V^{e x t}$. Since $\delta n=|e|^{-1} \vec{\nabla} \cdot \vec{p}$ and $\vec{E}^{e x t}=|e|^{-1} \vec{\nabla} V^{e x t}$ it follows that in general ${ }^{53},{ }^{54}$

$$
\chi\left(\vec{r}, \vec{r}^{\prime}, \omega\right)=-e^{-2} \partial_{i} \partial_{j^{\prime}} \alpha_{i j}^{s c r}\left(\vec{r}, \vec{r}^{\prime} \omega\right)
$$

Note that this "screened" or "interacting " polarizability $\alpha^{s c r}$ (denoted $-e^{-2} F$ in ref. 53) gives the response of the polarization to the external field whereas the usual polarizability $\alpha \equiv(\varepsilon-1) / 4 \pi$ gives the response to the total field. Thus $\alpha$ is an "irreducible" quantity that does not describe the RPA-level screening effects within a layer, in contrast to $\alpha^{s c r}$. Applying (9) to (6) and (8) we find via integration by parts (where henceforth we assume a maximally symmetric layer where the polarizability decouples into an $x x \equiv y y \equiv \|$ component and a $z z \equiv \perp$ component)

$$
\begin{aligned}
& R_{I}(\vec{Q}, \omega)=-2 \pi Q\left(\alpha_{x x}^{2 D, s c r,+}(Q, \omega)+\alpha_{z z}^{2 D, s c r,+}(Q, \omega)\right) \\
& T_{I}(\vec{Q}, \omega)=-2 \pi Q\left(\alpha_{x x}^{2 D, s c r,-}(Q, \omega)-\alpha_{z z}^{2 D, s c r,-}(Q, \omega)\right)
\end{aligned}
$$


where the screened layer polarizability of layer $I$ is

$$
\alpha_{i i}^{2 D, s c r, \pm}(Q, \omega)=\int_{\mathcal{R}_{I}} d z d z^{\prime} e^{Q\left(\left(z-Z_{I}\right) \pm\left(z^{\prime}-Z_{I}\right)\right)} \alpha_{i i}^{s c r}\left(\vec{Q}, z, z^{\prime}, i u\right)
$$

Here the four quantities $\alpha_{i i}^{2 D, s c r, \pm}(Q, \omega)$ for $i=x$ or $z$ are the lumped screened polarizabilities of a single layer, and in the limit $Q \rightarrow 0$ they all reduce to the polarization per unit area of the layer per unit external electric field $E^{e x t}$. For a layer with inversion symmetry in the $z$ direction, with $Z_{I}$ chosen at the symmetry point, an expansion in powers of $Q$ shows that the "+" and "-" versions $\alpha_{i i}^{2 D, s c r, \pm}(Q, \omega)$ are the same through $O(Q)$. Thus in $(10)$ and $(11)$ we can drop the superscripts+ and - on the polarizabilities $\alpha$ in many of the applications below.

An advantage of using (10) and (11) is that, for an insulating/semiconducting layer, $\alpha_{i i}^{2 D, s c r \pm}$ approach finite nonzero constants $\alpha_{x x}^{s c r}(\omega)$ and $a_{z z}^{s c r}(\omega)$ as $Q \rightarrow 0$, so that $R$ and $T$ vanish linearly with $Q$.

For a metallic layer or graphene, $\alpha_{x x}^{s c r}(\omega)$ diverges as $\omega \rightarrow 0$ and special care must be taken.

\section{MOMENT RESPONSE OF A SINGLE LAYER}

When an external potential of form (3) is applied to layer $I$, we find from (4) that the moments defined in (2) are

$$
\begin{aligned}
& m_{I}^{+}=\frac{Q}{2 \pi e^{2}}\left(R_{I}^{r i g h t} V_{I}^{+}+T_{I}^{l e f t} V_{I}^{-}\right) \\
& m_{I}^{-}=\frac{Q}{2 \pi e^{2}}\left(T_{I}^{r i g h t} V_{I}^{+}+R_{I}^{l e f t} V_{I}^{-}\right)
\end{aligned}
$$

This can be written

$$
\vec{m}_{I}=\frac{Q}{2 \pi e^{2}} \mathbf{c}_{0 I} \vec{V}_{I}=\frac{Q}{2 \pi e^{2}} \sum_{J} \mathbf{c}_{0 I J} \vec{V}_{J}
$$

where $\vec{m}_{I}=\left(m_{I}^{+}, m_{I}^{-}\right)^{T}$ and $\vec{V}_{I}=\left(V_{I}^{+}, V_{I}^{-}\right)^{T}$ are $2 \mathrm{D}$ column vectors and

$$
\mathbf{c}_{0 I}=\left(\begin{array}{ll}
R_{I}^{\text {right }} & T_{I}^{\text {left }} \\
T_{I}^{\text {right }} & R_{I}^{\text {left }}
\end{array}\right), \mathbf{c}_{0 I J}=\delta_{I J}\left(\begin{array}{ll}
R_{I}^{\text {right }} & T_{I}^{\text {left }} \\
T_{I}^{\text {right }} & R_{I}^{\text {left }}
\end{array}\right)
$$

is a dimensionless response function for the single isolated layer. We note that even for a non-symmetric layer the general reciprocity relation $\chi\left(\vec{r}, \vec{r}^{\prime}, i u\right)=\chi\left(\vec{r}^{\prime}, \vec{r}, i u\right)$ implies that $T^{\text {left }}(\vec{Q}, i u)=T^{\text {right* }}(\vec{Q}, i u)$ so that $\mathbf{c}_{0 I}$ is a hermitian matrix. (But there is in general no simple relation between $R^{\text {right }}$ and $R^{\text {left }}$ for a non-symmetric layer).

\section{RPA EQUATION FOR RESPONSE OF INTERACTING LAYERS}

Here the electron-electron Coulomb interactions within a layer are assumed to be included already in the layer response quantities $R_{I}$ and $T_{I}$. We now treat the Coulomb interactions between the layers in the Random Phase Approximation (RPA, time-dependent Hartree approximation). This means that the potential coefficients $V_{I}^{ \pm}$acting on layer $I$ in (13) consist of an externally imposed potential $V_{I, \text { ext }}^{ \pm}$and an internal potential $V_{I, \text { int }}^{ \pm}$that is generated by the dynamic charge moments $m_{J}^{ \pm}, J \neq I$, existing on the other layers, as in (2):

$$
V_{I, \text { int }}^{+}=\sum_{J>I} \int_{z^{\prime} \in R_{J}} d z^{\prime} \frac{2 \pi e^{2}}{Q} e^{-Q\left|Z_{I}-\left(Z_{J}+z^{\prime}\right)\right|} \delta n_{J}\left(z^{\prime}\right)=\frac{2 \pi e^{2}}{Q} \sum_{J} v_{I J}^{+-} m_{J}^{-}
$$

and similarly $V_{I, \text { int }}^{-}$is sourced from layers to the left, with $J<I$. These two cases can be summarized by

$$
\vec{V}_{I, i n t}=\frac{2 \pi e^{2}}{Q} \sum_{J} \mathbf{v}_{I J} \vec{m}_{J}
$$

where we have defined a $2 \times 2$ dimensionless interlayer Coulomb matrix by

$$
\mathbf{v}_{I J}=\equiv\left(\begin{array}{cc}
v_{I J}^{++} & v_{I J}^{+-} \\
v_{I J}^{-+} & v_{I J}^{-}
\end{array}\right)=e^{-Q\left|Z_{I}-Z_{J}\right|}\left(\begin{array}{cc}
0 & \bar{\theta}_{J>I} \\
\bar{\theta}_{I>J} & 0
\end{array}\right)
$$


where $\bar{\theta}_{\text {cond }}=1$ if the condition cond is satisfied, and equals 0 otherwise. Then the RPA equations are (with a Coulomb reduction factor $\lambda$ that equals 1 for the real system)

$$
\vec{m}_{I}=\vec{m}_{I}^{e x t}+\mathbf{c}_{0 I} \lambda \sum_{J} \mathbf{v}_{I J} \vec{m}_{J}
$$

Here, in the source term

$$
m_{I}^{ \pm e x t}=\int \exp ( \pm Q z) \chi_{I}\left(Q, z, z^{\prime}, i u\right) V^{e x t}\left(z^{\prime}\right) d z d z^{\prime}
$$

we have assumed no particular $z$ dependence of the external potential $V^{e x t}\left(z^{\prime}\right) \exp (\vec{Q} \cdot \vec{r}--i \omega t)$. If indeed the external potential is of the form (3) then $\vec{m}_{I}^{e x t}=\frac{Q}{2 \pi e^{2}} \mathbf{c}_{0 I} \vec{V}_{I}^{e x t}$ and so

$$
\vec{m}_{I}=\frac{Q}{2 \pi e^{2}} \mathbf{c}_{0 I} \vec{V}_{I}^{t o t}=\mathbf{c}_{0 I}\left(\frac{Q}{2 \pi e^{2}} \vec{V}_{I}^{e x t}+\lambda \sum_{J} \mathbf{v}_{I J} \vec{m}_{J}\right)
$$

The equations are off-diagonal in the layer index $I$ and have the formal solution

$$
\begin{gathered}
\vec{m}_{I}=\frac{Q}{2 \pi e^{2}} \sum_{J} \mathbf{c}_{\lambda I J} \vec{V}_{J}^{e x t} \\
\mathbf{c}_{\lambda}=\left(\mathbf{I}-\lambda \mathbf{c}_{0} \mathbf{v}\right)^{-1} \mathbf{c}_{0}
\end{gathered}
$$

where the inverse is over both the $I, J$ (layer) and +- (reflection / transmision) indices. ( $\mathbf{c}_{\lambda}$ is the response of the multi-layer system with both intra- and inter-layer Coulomb interactions included).

\section{PRACTICAL DETERMINATION OF $\alpha^{2 D, s c r}$ FOR $Q \rightarrow 0$}

The long-wavelength $2 \mathrm{D}$ screened polarizability $\alpha^{2 D, s c r}$ of a single isolated layer from (12) will be used as input for much of what follows. While there exist intrinsically $2 \mathrm{D}$ codes (such as ADF Band) and other approaches ${ }^{13}$ that can calculate $\alpha^{2 D, s c r}$ (or the correponding density response) directly, most condensed matter calculations use 3D planewave codes such as ABINIT or VASP ${ }^{49},{ }^{50}$. We show here that we can obtain $\alpha^{2 D, s c r}(Q \rightarrow 0, i \omega)$ from such $3 \mathrm{D}$ codes by finding the 3D macroscopic dielectric function of an infinite stack of parallel layers, with a layer spacing $D$ that only modestly exeeds the equilibrium spacing, $D \geq 2 D_{0}$. Of course the single-layer properties could be deduced by choosing a very large $D$ value, but such calculations are numerically prohibitive because of the large unit cell involved. Instead we show how to deconvolute the layer-layer Coulomb interactions, so the result for $\alpha^{2 D, s c r}$ converges as soon as $D$ is large enough to avoid overlap of layers.

\section{A. Response of a homogeneous stack of layers to a uniform field directed parallel to the layers.}

Consider an infinite stack of identical symmetric non-overlapping layers with an equal interlayer spacing $D \neq D_{0}$ that is not necessarily equal to the equilibrium crystal spacing $D_{0}$. We aim to relate the screened polarizability $\alpha_{x x}^{2 D}(Q, \omega)$ of a single isolated layer to the macroscopic dielectric properties of the stack. To simulate linear response to a uniform field $E_{0}$ in the $x$ direction, we choose

$$
V^{e x t}(\vec{r})=+E_{0}|e| x=E_{0}|e| \lim _{Q \rightarrow 0} \frac{\sin (Q x)}{Q}=E_{0}|e| \lim _{Q \rightarrow 0} \frac{e^{i Q x}-e^{-i Q x}}{2 i Q}
$$

Since there is no component of the external field in the $\mathrm{z}$ direction, this is independent of $\mathrm{z}$. Since for a symmetric layer such as graphene or BN the long-wavelength density response is even in $\vec{Q}$, this field induces a density of the same sinusoidal form, plus more rapidly varying local-field contributions:

$$
\delta n(\vec{r})=E_{0}|e| \frac{\sin (Q x)}{Q} \lim _{Q \rightarrow 0} \delta n(Q, z)+\{\vec{G} \neq \overrightarrow{0} \text { terms }\}
$$


where $\delta n(Q, z)=\int \tilde{\chi}\left(\vec{Q}, z, z^{\prime}\right) d z^{\prime}$ is the response to a unit potential $\exp (i \vec{Q} \cdot \vec{r})$ and $\tilde{\chi}$ is here the density response with all layer-layer interactions included. Because of the sum rules for density response ${ }^{53}, \tilde{\chi}$ can be derived from a well-behaved polarizability $\tilde{\alpha}$ as per Eq (9). The dipole moment $\vec{p}$ per unit volume, of the stack, in the $x$ direction can be found by looking at the polarization, specifically the dipole moment induced in a spatial region one half-wavelength $\lambda / 2=\pi / Q$ long in the $x$ direction, one layer (say the $I=0$ layer) wide in the $\mathrm{z}$ direction, and of unit width in the $y$ direction. The polarization induced by a strictly uniform field is a tricky concept and in reality is related to surface charges. Its calculation from the density perturbation in a finite region inside the crystal is well known to be subject to ambiguities: the answer depends on choice of the "edges" of the region. Equivalently, the net dipole moment depends strongly on the edge positions via the high-wavenumber $(\vec{G} \neq \overrightarrow{0})$ components of the induced electron density.

However, for an external field with a small but finite wavenumber $Q=2 \pi / \lambda$, considering a region $-\lambda / 4<x<\lambda / 4$, we find that the field $E_{0} \cos (Q x)$ from (21) vanishes at the edges. Correspondingly, explicit integration over this region shows that the dipole moment from the $\vec{G} \neq \overrightarrow{0}$ components of the induced density (22) contributes a zero fraction of the total moment as $Q \rightarrow 0$. Thus the dipole moment $\vec{p}$ per unit volume can be calculated from just the $\vec{G}=\overrightarrow{0}$ density components, as we do here in deriving Eqs (30) and (31) below.

Then the dipole moment per unit volume is

$$
\begin{aligned}
\vec{p} & =\frac{1}{1(\lambda / 2) D} \sum_{I} \int_{-\lambda / 4}^{+\lambda / 4} d x x \int_{-D / 2}^{+D / 2} d z(-|e| \delta n(\vec{r})) \\
& =\frac{-E_{0}|e|^{2}}{1(\pi / Q) D}\left(\int_{-\pi /(2 Q)}^{\pi /(2 Q)} x \frac{\sin (Q x)}{Q} d x\right) \int d z d z^{\prime} \tilde{\chi}\left(Q, z, z^{\prime}, \omega\right)
\end{aligned}
$$

From (9) the moment integral here is

$$
\begin{aligned}
\int d z d z^{\prime} \tilde{\chi}\left(Q, z, z^{\prime}, \omega\right) & =\int\left(Q^{2} \tilde{\alpha}_{\|}\left(Q, z, z^{\prime}, \omega\right)-\frac{\partial^{2}}{\partial z \partial z^{\prime}} \tilde{\alpha}_{\perp}\left(Q, z, z^{\prime}, \omega\right)\right) d z d z^{\prime} \\
& =Q^{2} \int \tilde{\alpha}_{\|}\left(Q, z, z^{\prime}, \omega\right) d z d z^{\prime}
\end{aligned}
$$

and this is not identical to the moments $m^{ \pm}$introduced in (2). However for small finite $Q$ the combination

$$
\begin{aligned}
\frac{1}{2}\left(m^{+}+m^{-}\right) & =\int \cosh (Q z) \delta n(Q, z, \omega) d z \\
& =\int\left(1+\frac{1}{2} Q^{2} z^{2}+\ldots\right) \tilde{\chi}\left(Q, z, z^{\prime}, \omega\right) d z d z^{\prime} \\
& =\int\left(1+\frac{1}{2} Q^{2} z^{2}+\ldots\right)\left(Q^{2} \tilde{\alpha}_{\| \mid}\left(Q, z, z^{\prime}, \omega\right)-\frac{\partial^{2}}{\partial z \partial z^{\prime}} \tilde{\alpha}_{\perp}\left(Q, z, z^{\prime}, \omega\right)\right) d z d z^{\prime} \\
& =Q^{2} \int \tilde{\alpha}_{\| \mid}\left(Q, z, z^{\prime}, \omega\right) d z d z^{\prime}+0+\frac{1}{2} Q^{4} \int z^{2} \tilde{\alpha}_{\|}\left(Q, z, z^{\prime}, \omega\right)+0
\end{aligned}
$$

gives the required moment (24) to lowest order in $Q$.Then (23) becomes to leading order in $Q$

$$
\vec{p}=\frac{-E_{0} e^{2} \hat{x}}{1(\pi / Q) D}\left(2 / Q^{3}\right) \frac{1}{2}\left(m^{+}+m^{-}\right) .
$$

Here we used $\int_{-\pi / 2}^{\pi / 2} x \sin x d x=2$ so $\int_{-\pi / q}^{\pi / q} q^{-1} x \sin (q x) d x=2 / q^{3}$ :

For this external potential (21) the external source term (17) in (16) is, with $\chi_{I}$ the density response of an isolated layer and arguments $Q, \omega$ suppressed in places for brevity,

$$
\begin{aligned}
m^{ \pm e x t} & =\int \exp ( \pm Q z) \chi_{I}\left(Q, z, z^{\prime}, i u\right) d z d z^{\prime} \\
& =\int \exp ( \pm Q z)\left(-e^{-2}\right)\left(Q^{2} \alpha_{\|}\left(z, z^{\prime}\right)-\frac{\partial^{2}}{\partial z \partial z^{\prime}} \alpha_{\perp}\left(z, z^{\prime}\right)\right) d z d z^{\prime} \\
& =-e^{-2} Q^{2} \int \exp ( \pm Q z) \alpha_{\|}\left(z, z^{\prime}\right) d z d z^{\prime} \quad \sin c e \int \frac{\partial^{2}}{\partial z \partial z^{\prime}} \alpha_{\perp}\left(z, z^{\prime}\right) d z^{\prime} \equiv 0 \\
& =-e^{-2} Q^{2} \alpha_{\|}^{2 D, s c r}(Q=0)+O\left(Q^{4}\right) \quad \text { for insulating layers } .
\end{aligned}
$$


For this z-independent source potential the density perturbation $m_{I}^{ \pm}$and the response matrix $\mathbf{c}_{0}$ are the same for any layer, $m_{I}^{ \pm}=m^{ \pm}$and then (16) becomes, to lowest order in $Q$

$$
\begin{aligned}
& \vec{m}=-e^{-2} Q^{2} \alpha_{\|}^{2 D, s c r}(Q=0)\left(\begin{array}{l}
1 \\
1
\end{array}\right)+\mathbf{c}_{0} \sum_{J} \mathbf{v}(I-J) \vec{m} \\
& \vec{m}=-e^{-2} Q^{2} \alpha_{\|}^{2 D, s c r}(Q=0)\left(\begin{array}{l}
1 \\
1
\end{array}\right)+\left(\begin{array}{cc}
T v & R v \\
R v & T v
\end{array}\right) \vec{m}
\end{aligned}
$$

where

$$
v=\sum_{K=1}^{\infty} e^{-K Q D}=\sum_{K=-1}^{-\infty} e^{K Q D}=\frac{1}{e^{Q D}-1} \approx \frac{1}{Q D} \text { for } Q \rightarrow 0
$$

Defining $m=\frac{1}{2}\left(m^{+}+m^{-}\right)$and adding the + and - components of $(26)$ we find

$$
\begin{aligned}
2 m & =-2 e^{-2} Q^{2} \alpha_{\|}^{2 D, s c r}(Q=0)+2(T+R) v m \\
m & =\frac{-e^{-2} Q^{2} \alpha_{\|}^{2 D, s c r}(Q=0)}{1-(T+R) \frac{1}{Q D}}=\frac{-e^{-2} Q^{2} \alpha_{\|}^{2 D, s c r}(Q=0)}{1+4 \pi D^{-1} \alpha_{\|}^{2 D, s c r}(Q=0)}
\end{aligned}
$$

The dipole moment per unit volume from (25) is then

$$
\vec{p}=\frac{2}{\pi} \frac{E_{0} \hat{x}}{D} \frac{\alpha_{\|}^{2 D, s c r}(Q=0)}{1+4 \pi D^{-1} \alpha_{\|}^{2 D, s c r}(Q=0)}
$$

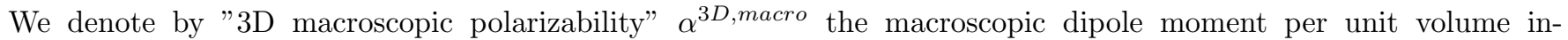
duced per unit external field, $\alpha^{3 D, \text { macro }}=p / \bar{E}$. Since the average external field in our chosen volume is $\bar{E}=\int_{-\pi /(2 Q)}^{\pi /(2 Q)} E_{0} \cos (Q x) d x / \int_{-\pi /(2 Q)}^{\pi /(2 Q)} d x=2 E_{0} / \pi$ we have from (28)

$$
\alpha^{3 D, \text { macro }}=\frac{p}{\bar{E}}=\frac{D^{-1} \alpha_{\|}^{2 D, s c r}(Q=0)}{1+4 \pi D^{-1} \alpha_{\|}^{2 D, s c r}(Q=0)}
$$

However the 3D macroscopic polarizability can also be expressed in terms of the (3D) macroscopic dielectric function $\varepsilon^{\text {macro }}$ of the stack, via $\alpha^{3 D \text {,macro }}=(4 \pi)^{-1}\left(1-1 / \varepsilon^{\text {macro }}\right)$. By equating this to (29) we find an expression for the polarizability of an isolated layer, valid when the layers do not overlap.

$$
\alpha_{\|}^{2 D, s c r}(Q=0, \omega)=\frac{D}{4 \pi}\left(\varepsilon_{\|}^{\text {macro }}(D, \omega)-1\right)
$$

This equation can alternatively be obtained via the type of analysis leading to (43) below, with $q_{z}=0$ exactly and $Q$ small but finite. Eq (30) is also implicit in the work of Nazarov ${ }^{51}$.

\section{B. Response of a homogeneous stack of layers to a uniform field directed perpendicular to the layers.}

For the case of a layer with inversion symmetry in the $x y$ directions, a uniform field in the $z$ direction (i.e. with $Q=0)$ can only move the $\vec{G}=\overrightarrow{0}$ component of charge in each layer of the stack in the $z$ direction, creating a perturbation charge on each layer that sums to zero and is zero outside the layer. By Gauss's theorem, such a charge distribution produces zero electric field outside the layer. Therefore the layers do not interact in this geometry provided that the spacing $D$ is stretched far enough from equilibrium that the layer charge densities do not overlap. Therefore the polarization per unit volume in the bulk is just obtained by adding the polarizations of the layers, traeated as independent, to the same external field. The polarization per unit volume in bulk in terms of the macroscopic dielectric function is

$$
a_{z z}^{3 D, s c r}=\frac{1}{4 \pi}\left(1-\left(\varepsilon_{r p a}^{-1}(q \hat{z}, q \rightarrow 0)\right)_{00}\right)=\frac{1}{4 \pi}\left(1-\frac{1}{\varepsilon_{z z}^{m a c r o}}\right)
$$


and for mutually non-interacting layers this must equal $\alpha_{z z}^{2 D, s c r} / D$. Thus

$$
\alpha_{z z}^{2 D, s c r}(Q=0, \omega)=\frac{D}{4 \pi}\left(1-\frac{1}{\varepsilon_{z z}^{\text {macro }}(D, \omega)}\right)
$$

Contrast this with the parallel-field case (30) where the macroscopic dielectric constant $\varepsilon$ appears in different way because of interactions between the layers in the parallel-field case. The result (31) can be derived more formally along the same lines as for (30), by using the type of analysis leading to (43), then setting $Q=0$ exactly while $q_{z}$ is small but finite. Eq (31) can also be obtained from equations in ref. 51 and is related to the work of Andersen et al ${ }^{52}$.

As an example of the formulae (31) and (30), Figs 2(a) and 3(a) show the parallel and perpendicular layer polarizabilities $\operatorname{Im}\left\{\alpha_{x x}^{2 D}\right.$, scr $\left.(\omega+i 0)\right\}$ and $\operatorname{Im}\left\{\alpha_{z z}^{2 D}\right.$,scr $\left.(\omega+i 0)\right\}$ of a BN monolayer, deduced from VASP calculations of the macroscopic dielectric functions of solid BN that is only slightly stretched. The agreement for layer spacings $D=0.8 \mathrm{~nm}$ and $D=1.2 \mathrm{~nm}$ shows that, with the analytic interlayer-descreening implied in (30), large layer spacings are not needed to extract well-converged properties of an isolated layer. On the other hand, if the "noninteracting" formula (31) is inappropriately applied to the parallel polarizability, the the results are strongly $D$-dependent (see Fig 2(b) ) and will only converge to the correct layer polarizability when $D \rightarrow \infty$.

As a further test we note that the BN binding energies and asymptotics that we obtain below are stable to $1 \%$ when we change from $D=0.8$ to $D=1.2 \mathrm{~nm}$ for the reference solid, in calculations of $\varepsilon^{\text {macro }}$.

\section{INTER-LAYER CORRELATION ENERGY IN ACFD-RPA}

For a collection of parallel non-overlapping layers we can separate the Coulomb interaction into intra-layer and inter-layer parts:

$$
V=V_{\text {intra }}+\lambda V_{\text {inter }}
$$

with $0 \leq \lambda \leq 1: \lambda=1$ defines the physical system, while for $\lambda=0$ we have a collection of isolated systems. We start from the full interaction $V_{\text {intra }}$ inside each layer, and turn on the interlayer interactions by varying $\lambda$ from 0 to 1 . By evaluating the work to increase from $\lambda$ to $\lambda+d \lambda$ we find the interlayer correlation energy in the Adiabatic Connection Fluctuation (ACFD) approach $^{48}$ :

$$
E^{\text {interlayer }}=\frac{\hbar}{2 \pi} \int_{0}^{\infty} d u \int_{r, r^{\prime} \text { in different layers }} d \vec{r} d \vec{r}^{\prime} \int_{0}^{1} d \lambda \chi_{\lambda}\left(\vec{r}, \vec{r}^{\prime}, i u\right) \frac{e^{2}}{\left|\vec{r}-\vec{r}^{\prime}\right|}
$$

Here $\chi_{\lambda}$ is the density-density response with the interaction (32) present. Fourier transformation of the $x$ and $y$ coordinates, with neglect of the exponentially decaying $\vec{G} \neq \overrightarrow{0}$ terms, gives the energy per unit area as

$$
\begin{aligned}
\frac{E^{\text {interlayer }}}{A}= & \frac{\hbar}{(2 \pi)^{3}} \int_{0}^{\infty} d u \int d^{2} Q \sum_{I \neq J} e^{-Q\left|Z_{I}-Z_{J}\right|} \frac{2 \pi e^{2}}{Q} \int_{0}^{1} d \lambda \\
& \times \int d Z d Z^{\prime} e^{\theta\left(Z_{J}-Z_{I}\right) Q\left(Z-Z^{\prime}\right)} \chi_{\lambda}\left(Q, Z_{I}+Z, Z_{J}+Z^{\prime}, i u\right)
\end{aligned}
$$

where $A$ is the area of one layer and $\theta$ is the Heaviside function. Here for $J>I$ the $\int d Z d Z^{\prime}$ can be recognized as the moment $m_{I}^{+}(\mathrm{Eq}(2))$ induced on layer $I$ by a perturbing external potential component $V_{J}^{-}$of unit strength (see Eq (3)) applied to layer $J$ only. This moment would be zero if the layers did not interact. For interacting layers, $\vec{m}$ is obtained from solution of (18) with $\vec{V}_{I}^{e x t}=\left(\delta_{I J}, 0\right)$. Similarly for $J<I$ the integral $\int d z d z^{\prime}$ gives $m_{I}^{-}$for a unit source $V^{-}$in layer $J$, so that $\vec{V}_{I}^{e x t}=\left(0, \delta_{I J}\right)$. Thus from (19)

$$
\int d z d z^{\prime} e^{\theta(J-I) Q\left(z-z^{\prime}\right)} \chi\left(Q, Z_{I}+z, Z_{J}+z^{\prime}, i u\right)=\frac{Q}{2 \pi e^{2}}\left(c_{I J}^{+-} \theta(J-I)+c_{I J}^{-+} \theta(I-J)\right)
$$

and then (33) with (15) becomes

$$
\begin{aligned}
\frac{E^{\text {interlayer }}}{A} & =\frac{\hbar}{(2 \pi)^{3}} \int_{0}^{\infty} d u \int d^{2} Q \sum_{I J} \int_{0}^{1} d \lambda\left(c_{\lambda I J}^{+-} v_{J I}^{-+}+c_{\lambda I J}^{+-} v_{J I}^{-+}\right) \\
& =\frac{\hbar}{(2 \pi)^{3}} \int_{0}^{\infty} d u \int d^{2} Q \sum_{I J} \int_{0}^{1} d \lambda T r_{ \pm}\left(\mathbf{c}_{\lambda I J} \mathbf{v}_{J I}\right)
\end{aligned}
$$


where $\mathbf{v}_{I J}$ is defined in (15). Here the trace $\mathbf{T} r_{ \pm}$is over a $2 \times 2$ matrix but the layer sum in (34) can also be recognized as a trace of the product $\mathbf{c v}$ over the layer index $I$. Expanding (20) to zero order in the interlayer interaction $u$ and noting $\mathbf{c}_{0}=\delta_{I J} \mathbf{c}_{0 I I}$ we find that the lowest-order contribution to (34) is zero, being proportonal to $\mathbf{T} r_{ \pm} \sum_{I} \mathbf{c}_{0 I I} v_{I I}=0$ since $\mathbf{v}_{I I}=\mathbf{0}$ from (15). The expansion of (34) therefore starts at second order in $\mathbf{v}$. The second order term is proportional to a sum of interactions between pairs $I, J$, of layers, $\sum_{I J} \int \lambda d \lambda \operatorname{Tr}\left(\mathbf{c}_{0 I I} \mathbf{v}_{I J} \mathbf{c}_{0 J J} \mathbf{v}_{J I}\right)$. Similar arguments show that expansion terms in (34) containing solely $v^{+}$factors, or solely $v^{-}$factors, are also zero these terms couple only layers to the right(left) of each other and so cannot contribute to the same-layer components that make up the trace.

\section{CORRELATION ENERGY BETWEEN TWO PARALLEL LAYERS DISTANT $D$}

Here we set the layer spacing $Z_{2}-Z_{1}=D$. and (15) becomes

$$
\mathbf{v}_{\mathbf{1 2}}=\left(\begin{array}{cc}
0 & \exp (-Q D) \\
0 & 0
\end{array}\right)=\mathbf{v}_{\mathbf{2 1}}^{T}
$$

Here it is appropriate to drive with $V_{1}^{-}=V_{2}^{+}=0$, so that only two of the four scalar RPA equations (18) are coupled:

$$
\begin{gathered}
\vec{m}_{I}=\frac{Q}{2 \pi e^{2}} \mathbf{c}_{0 I} \vec{V}_{I}^{t o t}=\mathbf{c}_{0 I}\left(\frac{Q}{2 \pi e^{2}} \vec{V}_{I}^{e x t}+\lambda \sum_{J} \mathbf{v}_{I J} \vec{m}_{J}\right) \\
m_{1}^{+}=c_{01}^{++}\left(\frac{Q}{2 \pi e^{2}} V_{1}^{+}+\lambda e^{-Q D} m_{2}^{-}\right) \\
m_{2}^{-}=c_{02}^{--}\left(\frac{Q}{2 \pi e^{2}} V_{2}^{-}+\lambda e^{-Q D} m_{1}^{+}\right)
\end{gathered}
$$

with solution

$$
m_{1}^{+}=\frac{Q}{2 \pi e^{2}} \frac{c_{01}^{++} V_{1}^{+}+\lambda e^{-Q D} c_{01}^{++} c_{02}^{--} V_{2}^{-}}{\left(1-\lambda^{2} e^{-2 Q D} c_{01}^{++} c_{02}^{--}\right)}=\frac{Q}{2 \pi e^{2}}\left(c_{11}^{++} V_{1}^{+}+c_{12}^{+-} V_{2}^{-}\right)
$$

so that the interacting response function between the layers is

$$
c_{12}^{ \pm}=\frac{\lambda e^{-Q D} c_{01}^{++} c_{02}^{--}}{1-\lambda^{2} e^{-2 Q D} c_{01}^{++} c_{02}^{--}}=c_{21}^{\mp}
$$

Then the first line of (34) becomes

$$
\begin{aligned}
\frac{E^{\text {interlayer }}}{A} & =\frac{\hbar}{(2 \pi)^{3}} \int_{0}^{\infty} d u \int d^{2} Q \int_{0}^{1} d \lambda\left(c_{\lambda I J}^{+-} v_{J I}^{-+}+c_{\lambda I J}^{-+} v_{J I}^{+-}\right) \\
\frac{E^{\text {interlayer }}}{A} & =\frac{\hbar}{(2 \pi)^{3}} \int_{0}^{\infty} d u \int d^{2} Q \int_{0}^{1} d \lambda \frac{2 \lambda c_{02}^{--} c_{01}^{++} e^{-2 Q D}}{1-\lambda^{2} e^{-2 Q D} c_{01}^{++} c_{02}^{--}} \\
& =\frac{\hbar}{(2 \pi)^{3}} \int_{0}^{\infty} d u \int d^{2} Q \ln \left(1-e^{-2 Q D} c_{01}^{++} c_{02}^{--}\right) \\
& =\frac{\hbar}{(2 \pi)^{3}} \int_{0}^{\infty} d u \int d^{2} Q \ln \left(1-e^{-2 Q D} R_{1}^{\text {right }} R_{2}^{\text {left }}\right)
\end{aligned}
$$

where (14) was used.

We note in passing that $(37)$ is valid even for infinitely thick parallel slabs separated by distance $D$, where we choose the regions $\mathcal{R}_{1}$ and $\mathcal{R}_{2}$ to be $z<0$ and $z>0$ respectively, with the reference points $Z_{1}$ and $z_{2}$ at $\pm D / 2$ (i.e. at the edges of the two slabs). See Fig 1 . By evaluating the slab reflection coefficients $R_{1}$ and $R_{2}$ in the small-q limit (see for example ${ }^{48}$ ) one can then re-derive the electromagnetically non-retarded limit of the original Lifshitz result for non-overlapping slabs. Here however we consider nano-thin layers. 
For isotropic inversion-symmetric nano-layers, use of (10) gives

$$
\frac{E^{\text {interlayer }}}{A}=\frac{\hbar}{(2 \pi)^{3}} \int_{0}^{\infty} d u \int d^{2} Q \ln \left(1-e^{-2 Q D}(-2 \pi Q)^{2} \alpha_{1 t o t} \alpha_{2 t o t}\right)
$$

where for each layer species $\alpha_{t o t}=\alpha_{x x}^{2 D, s c r}(\vec{Q}, i u)+\alpha_{z z}^{2 D, s c r}(\vec{Q}, i u)$.

For large $D$ (at least for insulating layers) we can expand the logarithm in (38) to second order, giving

$$
\frac{E^{\text {interlayer }}}{A}=-\frac{\hbar}{(2 \pi)^{3}} \int_{0}^{\infty} d u \int(2 \pi Q)^{2} d^{2} Q e^{-2 Q D} \alpha_{1 t o t} \alpha_{2 t o t}
$$

For the case of a BN bilayer our numerical work (see below) shows that the maximum value of $\alpha_{\text {tot }}$ (iu) is about $0.1 \mathrm{~nm}$, and since the maximum value of $Q^{2} \exp (-2 Q D)$ is $e^{-2} D^{-2}$, the nontrivial term under the logarithm in (37) is always less than about $(2 \pi)^{2} 10^{-2} e^{-2}(\mathrm{D} / \mathrm{nm})^{-2}=5.3 \times 10^{-2}(\mathrm{D} / \mathrm{nm})^{-2}$. Thus for any $\mathrm{D}>2 \mathrm{D}_{0}=0.7 \mathrm{~nm}$ this term is less than $\approx 5.3 \times 10^{-2}(.7)^{-2}=0.11$ and so expansion of the logarithm is justified $a$ priori for BN layers except very close to contact. In practice we found the expansion is suitable for correlation energy calculations even near to contact.

\section{A. Asymptotic vdW interaction of two insulating layers}

For two insulating layers $\alpha_{1 \text { tot }}$ and $\alpha_{2 t o t}$ are both constant as $Q \rightarrow 0$. The asymptotic $(D \rightarrow \infty$, implying $Q \rightarrow 0)$ $\mathrm{vdW}$ interaction (38) is then evaluated by taking each $\alpha_{\text {itot }}$ as independent of $Q$, and Taylor-expanding the logarithm in $(38)$. We this obtain the asymptotic $(D \rightarrow \infty)$ form

$$
\left.\frac{E^{\text {interlayer }}}{A}(2 \text { insulators })\right)=-\frac{3 \hbar}{8 D^{4}} \int_{0}^{\infty} \alpha_{1 t o t}(Q=0, i u) \alpha_{2 o t}(Q=0, i u) d u
$$

This is total correlation energy per unit area of one side of the double layer. The correlation energy per unit area per layer is then half of this:

$$
\frac{E^{\text {interlayer }}(2 \text { ins })}{N A}=-\frac{3 \hbar}{16 D^{4}} \int_{0}^{\infty} \alpha_{1 t o t}(Q=0, i u) \alpha_{2 t o t}(Q=0, i u) d u
$$

where $N=2$ and $\alpha_{\text {tot }}$ is defined just below Eq (38).

\section{INTER-LAYER RPA INTERACTIONS IN AN INFINITE UNIFORM STACK OF IDENTICAL SYMMETRIC LAYERS}

Here all layers are identically constructed. With reference to Fig 1, the Ith layer is centred on the reference point

$$
Z_{I}=I D, I=0, \pm 1, \pm 2, \ldots
$$

and the region $\mathcal{R}_{I}$ containing layer $I$ is

$$
\mathcal{R}_{I}=\{z:(I-1 / 2) D<z<(I+1 / 2) D\}
$$

Here $D$ is the common spacing between adjacent layers, which does not have to be the equilibrium spacing (e.g. the layered crystal could be stretched).

For this equally-spaced case all two-index layer functions such as $\mathbf{c}_{I J}$ are functions of $I-J$ only. The inverse over the layer indices $I, J$ in (20) then becomes analytic via a reverse Fourier Series transformation to a wavenumber variable $q_{z}$, instead of layer index $I$. For an arbitrary function $f_{I}$ we define

$$
f\left(q_{z}\right)=\sum_{J} e^{-i q_{z} D J} f_{J}, \quad f_{I}=\frac{D}{2 \pi} \int_{-\pi / D}^{\pi / D} d q_{z} f\left(q_{z}\right) e^{i q_{z} D I}
$$


and a convolution $X_{\Delta I} \equiv X_{I-J}=\sum_{K} F_{I-K} G_{K-J}$ has a transform $X\left(q_{z}\right)=F\left(q_{z}\right) G\left(q_{z}\right)$.

Then (20) becomes

$$
\mathbf{c}\left(Q, q_{z}\right)=\left(I-\mathbf{c}_{0}\left(Q, q_{z}\right) \mathbf{v}\left(Q, q_{z}\right)\right)^{-1} \mathbf{c}_{0}\left(Q, q_{z}\right)
$$

where the products and inverse are solely over $2 \times 2$ matrices. Here from (14) and (41)

$$
\mathbf{c}_{0}\left(Q, q_{z}\right)=\left(\begin{array}{cc}
R(Q, \omega) & T(Q, \omega) \\
T(Q, \omega) & R(Q, \omega)
\end{array}\right)
$$

is independent of $q_{z}$. Similarly from (15) and (41),

$$
\mathbf{v}\left(Q, q_{z}\right)=\left(\begin{array}{cc}
0 & v^{+}\left(Q, q_{z}\right) \\
v^{+}\left(Q, q_{z}\right)^{*} & 0
\end{array}\right)
$$

where

$$
v^{+}\left(Q, q_{z}\right)=\sum_{I>0} \exp \left(\left(-Q+i q_{Z}\right) D I\right)=\frac{e^{i q_{z} D} e^{-Q D}}{1-e^{i q_{z} D} e^{-Q D}}=\left(v^{-}\right)^{*}
$$

Then the interlayer correlation energy per unit area, per layer of the infinite stack $(N \rightarrow \infty)$ from $(34)$ is a convolution on $J$ :

$$
\begin{aligned}
\frac{E_{c}}{N A} & =\frac{\hbar}{(2 \pi)^{3}} \int_{0}^{\infty} d u \int d^{2} Q \frac{1}{N} \sum_{I} \int_{0}^{1} d \lambda \mathbf{T r}_{ \pm}\left(\mathbf{c}_{\lambda} \mathbf{v}\right)_{I I} \\
& =\frac{\hbar}{(2 \pi)^{3}} \int_{0}^{\infty} d u \int d^{2} Q \int_{0}^{1} d \lambda \frac{D}{2 \pi} \int d q_{z} \mathbf{T r}_{ \pm}\left[\left(1-\lambda \mathbf{c}_{0} \mathbf{v}\right)^{-1} \mathbf{c}_{0} \mathbf{v}\right]_{Q, q_{z} . i u} \\
& =\frac{\hbar}{(2 \pi)^{3}} \mathbf{T r}_{\vec{q}} \mathbf{T r}_{ \pm} \int_{0}^{\infty} \hbar d u \ln \pm\left(\mathbf{I}-\mathbf{c}_{0} \mathbf{v}\right) \\
& =\int_{0}^{\infty} \hbar d u \mathbf{T} r_{\vec{q}} \ln \operatorname{det}\left(\mathbf{I}-\mathbf{c}_{0} \mathbf{v}\right) \\
& =(2 \pi)^{-3} \operatorname{Tr}_{\vec{q}} \int_{0}^{\infty} \hbar d u \ln \left(1-2 T R e v^{+}+\left(T^{2}-R^{2}\right)\left|v^{+}\right|^{2}\right)
\end{aligned}
$$

Here $\mathbf{T} r_{\vec{q}}\{\} \equiv \int_{0}^{\infty} d^{2} Q \frac{D}{2 \pi} \int_{-\pi / D}^{\pi / D}\{\} d q_{z}$, while $\mathbf{T} r_{ \pm}$and $\ln _{ \pm}$are operations on the $2 \times 2$ matrices. In (46), the last step used (42) and (43)). From (43) we have

$$
\begin{aligned}
R e v^{+} & =\left(\cos \left(q_{z} D\right)-\exp (-Q D)\right) / b, \operatorname{Tr}^{+}=\left|v^{+}\right|^{2}=\exp (-Q D) /(2 b) . \\
b & =\cosh (Q D)-\cos \left(q_{z} D\right)
\end{aligned}
$$

Then the inter-layer correlation energy of the infinite stack is

$$
\frac{E_{c}}{N A}=(2 \pi)^{-3} \mathbf{T} r_{\vec{q}} \int_{0}^{\infty} \hbar d u \ln \left(1+\frac{\left(\exp (-Q D)-\cos \left(q_{z} D\right)\right) T+\exp (-Q D)\left(T^{2}-R^{2}\right)}{\cosh (Q D)-\cos \left(q_{z} D\right)}\right)
$$

where $T(\vec{Q}, i u)$ and $R(\vec{Q}, i u)$ are the reflection and transmission coefficients.

This can be written in dimensionless form using $\kappa_{\|}=Q D, \kappa_{z}=q_{z} D$ and applying (11) and (10):

$$
\begin{aligned}
\frac{E_{c}}{N A}= & (2 \pi)^{-3} D^{-2} \int_{\vec{\kappa}_{||}} d^{2} \kappa_{\|} \frac{1}{2 \pi} \int_{-\pi}^{\pi} d \kappa_{z} \int_{0}^{\infty} \hbar d u \\
& \times \ln \left(1+\frac{-2 \pi \kappa_{\|} D^{-1}\left(\exp \left(-\kappa_{\| \mid}\right)-\cos \left(\kappa_{z}\right)\right) \alpha_{d i f f}+\left(2 \pi \kappa_{\|}\right)^{2} D^{-2} \exp \left(-\kappa_{\| \mid}\right)\left(\alpha_{\text {sum }}^{2}-\alpha_{d i f f}^{2}\right)}{\cosh \left(\kappa_{\|}\right)-\cos \left(\kappa_{z}\right)}\right)
\end{aligned}
$$

Here $\alpha_{\text {diff }}=\alpha_{x x}^{2 D, s c r,-}\left(\kappa_{\|} D^{-1}, i u\right)-\alpha_{z z}^{2 D, s c r,-}\left(\kappa_{\|} D^{-1}, i u\right), \quad \alpha_{s u m}=\quad \alpha_{x x}^{2 D, s c r,+}\left(\kappa_{\| \mid} D^{-1}, i u\right)+$ $\alpha z z^{2 D, s c r,+}\left(\kappa_{||} D^{-1}, i u\right)$. 


\section{A. $\quad D \rightarrow \infty$ limit for an infinite stack of insulating layers}

In the large- $D$ limit (49) calls for the single-layer polarizabilities $\alpha_{\text {sum }}, \alpha_{\text {diff }}$ at very small parallel wavenumber $Q=\kappa_{\| \mid} D^{-1}$ where the cosh function on the denominator ensures that large $\kappa_{\|}$values are not relevant. For insulators these polarizabilities have finite values as $Q \rightarrow 0$, and then (49) shows explicitly that the argument of the logarithm is close to 1, justifying a Taylor expansion of the log. In fact the expansion of the logarithm is most transparently done from the original formula (46), giving

$$
\frac{E^{(2)}}{N A}=\frac{1}{(2 \pi)^{3}} \mathbf{T} r_{\vec{q}} \int_{0}^{\infty} \hbar d u\left[-T\left(v^{+}+v^{+*}\right)+\left(T^{2}-R^{2}\right) v^{+} v^{+*}-\frac{1}{2} T^{2}\left(v^{+2}+2 v^{+} v^{+*}+v^{+* 2}\right)\right]
$$

The terms containing only $v^{+}$, or only $v^{+*}$, and those containing only $v^{+2}$ or $v^{+* 2}$ are zero as explained following Eq (34). The terms containing $T$ cancel and the remaining lowest term involves only the reflection coefficient and is of second order in $\mathbf{v}$ :

$$
\begin{aligned}
\frac{E^{(2)}}{N A} & =\frac{-1}{(2 \pi)^{3}} \mathbf{T} r_{\vec{q}}\left(v^{+} v^{+*} \int_{0}^{\infty} \hbar d u R^{2}\right) \\
& =\frac{-1}{(2 \pi)^{3}} \int\left(\frac{D}{2 \pi} \int v^{+} v^{+*} d q_{z}\right)(2 \pi Q)^{2} d^{2} Q \int_{0}^{\infty} \alpha_{t o t}^{2}(i u) \hbar d u
\end{aligned}
$$

where $\alpha_{\text {tot }}$ is defined below Eq (38). Now the $q_{z}$ integral is a Fourier coefficient of a product and therefore gives the on-layer value of a layer convolution,

$$
\frac{D}{2 \pi} \int v^{+} v^{+*} d q_{z}=\sum_{J} v_{I J}^{+} v_{J I}^{-}=\sum_{K=1}^{\infty} e^{-2 K D Q}
$$

Then

$$
\begin{aligned}
\frac{E^{(2) \inf \text { stack }(D)}}{N A} & =\frac{-1}{(2 \pi)^{3}} \sum_{K=1}^{\infty} \int d^{2} Q e^{-2 K D Q}(2 \pi Q)^{2} \int_{0}^{\infty} \alpha_{\text {tot }}^{2}(Q, i u) \hbar d u \\
& =2 \sum_{K=1}^{\infty} \frac{E^{(2) \text { bilayer }}(K D)}{2 A}
\end{aligned}
$$

where (39) was used. This form, representing a pairwise sum over layers, results from our expansion of the logarithm in (46). The fully asymptotic $\mathrm{D} \rightarrow \infty$ result can be obtained for insulating layers by using the $Q=0$ value of the layer response. The $Q$ integral can then be done analytically for each value of $K$, giving the asymptotic (large- $D$ ) layer binding energy for a uniform stack of identical insulating layers:

$$
\frac{E}{N A}=-\frac{3}{8} D^{-4}\left(\sum_{K=1}^{\infty} K^{-4}\right) \int_{0}^{\infty} \hbar d u \alpha_{t o t}^{2}(Q=0, i u)
$$

where $\alpha_{\text {tot }}$ was defined following $\mathrm{Eq}(38)$. Note $\sum_{K=1}^{\infty} K^{-4} \approx 1.082$. The leading term in the infinite stack result (51) is twice the two-layer result (40), which makes sense because in the infinite stack each layer has two nearest-neighbour layers.

We can convert (51) to an energy per atom by multiplying by the area $A_{\text {atom }}$ per atom in a layer.

\section{SUB-ASYMPTOTIC THEORY: RESPONSE AND ENERGETICS FOR SMALL NON-ZERO $Q$}

The asymptotic results (40), (51) above were based (at least for insulating layers) on the layer polarizabilities at $Q=0$. Now we look at the response of an isolated layer for small but finite $Q$, which will allow us to consider the interactions of layers with objects at somewhat closer distances, but still not in the region of strong overlap of electronic clouds. (i.e. it gives sub-asymptotic results). To do this we need to consider the Coulomb interaction within a layer, which we do at the RPA level. For these intra-layer interactions we cannot a priori ignore the $\vec{G} \neq \overrightarrow{0}$ (local-field) Coulomb interaction effects. 
The bare (Kohn-Sham) and interacting density responses $\chi^{(0)}, \chi$ of a single periodic layer are related by the RPA equation

$$
\chi_{\vec{G} \vec{G}^{\prime}}\left(Q, \omega, z, z^{\prime}\right)=\chi_{\vec{G} \vec{G}}^{(0)}\left(Q, \omega, z, z^{\prime}\right)+\sum_{\vec{G} \prime \prime} \int d z^{\prime \prime} d z^{\prime \prime \prime} \chi_{\vec{G} \vec{G}^{\prime \prime}}^{(0)}\left(Q, z, z^{\prime \prime}, \omega\right) V_{\vec{G}^{\prime \prime}+\vec{Q}^{\prime}}\left(z^{\prime \prime}, z^{\prime \prime \prime}\right) \chi_{\vec{G}^{\prime \prime} \vec{G}^{\prime}}\left(Q, \omega, z^{\prime \prime \prime}, z^{\prime}\right)
$$

or more compactly $\chi=\chi^{0}+\chi^{0} * V * \chi$ where the $2 \mathrm{D}$ Coulomb potential is $V_{\vec{k}}\left(z, z^{\prime}\right)=2 \pi e^{2}|\vec{k}|^{-1} \exp \left(-|\vec{k}|\left|z-z^{\prime}\right|\right)$. We break this Coulomb interaction into a part $W^{\text {rap }}$ that varies rapidly in space even when $Q \rightarrow 0$, and a part $W^{(\text {slow })}$ that does not: $V=W^{\text {rap }}+W^{\text {slow }}$.

The rapid component contains the $\vec{G} \neq \overrightarrow{0}$ local-field terms for $Q=0$, and another part with a discontinuous $z$ derivative:

$$
\frac{W_{G}^{r a p}\left(z, z^{\prime}\right)}{2 \pi e^{2}}=\left(1-\delta_{\vec{G}, \overrightarrow{0}}\right) \frac{\exp \left(-|\vec{G}|\left|z-z^{\prime}\right|\right)}{|\vec{G}|}-\delta_{\vec{G}, \overrightarrow{0}}\left|z-z^{\prime}\right|
$$

The remaining slowly varying part is analytic at small $Q$ :

$$
\begin{aligned}
\frac{W_{\vec{G}}^{\text {slow }}\left(z, z^{\prime}\right)}{2 \pi e^{2}}= & \frac{V-W^{\text {rap }}}{2 \pi e^{2}} \\
= & \left(1-\delta_{\vec{G}, \overrightarrow{0}}\right)\left(\frac{\exp \left(-|\vec{G}+\vec{Q}|\left|z-z^{\prime}\right|\right)}{|\vec{G}+\vec{Q}|}-\frac{\exp \left(-|\vec{G}|\left|z-z^{\prime}\right|\right)}{|\vec{G}|}\right) \\
& +\delta_{\vec{G}_{00}, \overrightarrow{0}}\left(\frac{\exp \left(-Q\left|z-z^{\prime}\right|\right)}{Q}+\left|z-z^{\prime}\right|\right) \\
= & \delta_{\vec{G}_{00}, \overrightarrow{0}}\left(Q^{-1}+\frac{1}{2} Q\left(z-z^{\prime}\right)^{2}\right)+O\left(Q^{2}\right)
\end{aligned}
$$

We define a "rapid" single-layer response $\chi_{\vec{G} \vec{G}^{\prime}}^{r a p}\left(Q, z, z^{\prime}, \omega\right)$ that satisfies (52) with $W^{r a p}$ in place of the full Coulomb interaction $V$ :

$$
\chi^{r a p}=\chi^{0}+\chi^{0} * W^{r a p} * \chi^{r a p}
$$

and it then follows that the full RPA response $\chi$ from (52) exactly satisfies

$$
\chi=\chi^{\text {rap }}+\chi^{\text {rap }} * W^{\text {slow }} * \chi
$$

To study the subasymptotic interaction of the layer with other layers, we only need the component $\vec{G}=\vec{G}^{\prime}=\overrightarrow{0}$ of $\chi$. We consider this component of (56). Expressing $\chi^{r a p}$ and $\chi$ in terms of polarizabilities $\alpha^{r a p}, \alpha^{s c r}$ as in (9) we write the $\vec{G}=\vec{G}^{\prime}=\overrightarrow{0}$ component of the convolution on the RHS of (56) in the the form

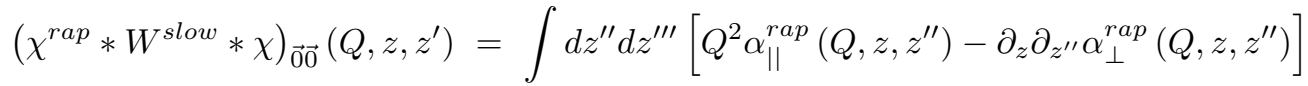

$$
\begin{aligned}
& \times\left(Q^{-1}+Q\left(z^{\prime \prime}-z^{\prime \prime \prime}\right)^{2}\right)\left[Q^{2} \alpha_{\|}^{s c r}\left(Q, z^{\prime \prime \prime}, z^{\prime}\right)-\partial_{z^{\prime \prime \prime}} \partial_{z^{\prime}} \alpha_{\perp}^{s c r}\left(Q, z^{\prime \prime \prime}, z^{\prime}\right)\right] \\
& =Q \int d z^{\prime \prime} d z^{\prime \prime \prime} \alpha_{\|}^{r a p}\left(Q, z, z^{\prime \prime}\right) Q^{2} \alpha_{\|}^{s c r}\left(Q, z^{\prime \prime \prime}, z^{\prime}\right)\left(1+O\left(Q^{2}\right)\right)
\end{aligned}
$$

where we used the identities $\int \partial_{z} \partial_{z^{\prime \prime}} \alpha_{\perp}^{r a p}\left(Q, z, z^{\prime \prime}\right) d z^{\prime \prime}=0=\int \partial_{z^{\prime \prime \prime}} \partial_{z^{\prime}} \alpha_{\perp}^{s c r}\left(Q, z^{\prime \prime \prime}, z^{\prime}\right) d z^{\prime \prime \prime}$. We take the $\exp \left(Q\left(z \pm z^{\prime}\right)\right) d z d z^{\prime}$ moments of (56) using (57) and also the definition (12), finding

$$
\begin{aligned}
Q^{2}\left(\alpha_{\|}^{2 D, s c r}(Q, i u) \pm \alpha_{\perp}^{2 D, s c r}(Q, i u)\right)= & Q^{2}\left(\alpha_{\|}^{2 D, r a p}(Q, i u) \pm \alpha_{\perp}^{2 D, r a p}(Q, i u)\right) \\
& +Q \alpha_{\|}^{2 D, r a p}(Q, i u) Q^{2} \alpha_{\|}^{2 D, s c r}(Q, i u)+O\left(Q^{4}\right)
\end{aligned}
$$

Here we have noted that the moments $\int \exp \left(Q\left(z \pm z^{\prime}\right)\right) \alpha_{i i}\left(Q, z, z^{\prime}\right) d z d z^{\prime}, \int \exp ( \pm Q z) \alpha_{i i}\left(Q z, z^{\prime}\right) d z d z^{\prime}$ are equal to to lowest order $\left(O\left(Q^{0}\right)\right)$, and also to $O\left(Q^{1}\right)$ for symmetric layers, which we consider exclusively here. Thus we were 
able to drop the \pm superscript on the $\alpha^{2 D}$ terms (see also the commentary following Eq (12)). The same is not true for the equivalent moments of $\chi$. Adding and subtracting the two equations (58) and cancelling $Q^{2}$ throughout, we find for a symmetric layer

$$
\begin{gathered}
\alpha_{\|}^{2 D, s c r}(Q, i u)=\alpha_{\|}^{2 D, r a p}(Q, i u)+2 \pi Q \alpha_{\|}^{2 D, r a p}(Q, i u) \alpha_{\|}^{2 D, s c r}(Q, i u)+O\left(Q^{2}\right) \\
\alpha_{\perp}^{2 D, s c r}(Q, i u)=\alpha_{\perp}^{2 D, r a p}(Q, i u)+O\left(Q^{2}\right)
\end{gathered}
$$

These screening equations are deceptively simple, as they include all one-electron local-field effects to the order

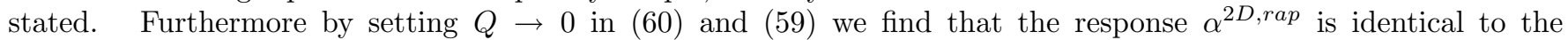
interacting layer response $\alpha^{2 D, s c r}$ in the $Q \rightarrow 0$ limit. Also, using the facts that $W^{\text {rap }}$ is $Q$-independent (see (53)) and $\chi^{0}(Q, i u)=\chi^{0}(Q \rightarrow 0)\left(1+O\left(Q^{2}\right)\right)$, we can show from $(55)$ that $\alpha^{2 D, \text { rap }}(Q, i u)=\alpha^{2 D, \text { rap }}(Q=0, i u)+O\left(Q^{2}\right)$. Thus (59) and (60) can be written, for a symmetric layer at least

$$
\begin{aligned}
& \alpha_{x x} \equiv \alpha_{\|}^{2 D, s c r}(Q, i u)=\frac{\alpha_{\|}^{2 D, s c r}(Q \rightarrow 0, i u)}{1+2 \pi Q \alpha_{\|}^{2 D, s c r}(Q \rightarrow 0, i u)}+O\left(Q^{2}\right), \\
& \alpha_{z z} \equiv \alpha_{\perp}^{2 D, s c r}(Q, i u)=\alpha_{\perp}^{2 D, s c r}(Q, \rightarrow 0, i u)+O\left(Q^{2}\right)
\end{aligned}
$$

where the $\alpha_{i i}{ }^{2 D, s c r}(Q \rightarrow 0, i u)$ are readily obtained from macroscopic dielectric function calculations as in (30) and (31).

We can use (61) and (62) in two ways, a formal next-order correction, and a less systematic approach that is more useful in practice.

\section{A. Formal next-order correction to inter-layer correlation energy}

We can expand (61) formally to 1st order, obtaining $\alpha_{\|}^{2 D, s c r}(Q, i u)=\alpha_{\|}^{2 D, s c r}(0, i u)-2 \pi Q\left[\alpha_{\|}^{2 D, s c r}(0, i u)\right]^{2}$. Putting this into (39), for example, we obtain a sub-asymptotic correction to (40) for the cross-correlation energy of two identical insulating layers

$$
E^{c, n e x t}=\frac{1}{D^{5}} \frac{3 \pi}{2} \hbar \int\left(\alpha_{\|}\right)^{2} \alpha_{t o t} d u
$$

where $\alpha_{t o t}=\alpha_{x x}^{2 D, s c r}(Q=0, i u)+\alpha_{z z}^{2 D, s c r}(Q=0, i u)$ and $\alpha_{\|}=\alpha_{x x}^{2 D, s c r}(Q=0, i u)$. Eq (63) is to be added to the leading $D^{-4}$ asymptotic term from (40). For an infinite stack, according to (50), (63) should be multiplied by $2 \sum_{J=1}^{\infty} J^{-5}=2.0738$. Surprisingly, we found that for BN layers, (63) becomes comparable to the leading asymptotic term (40) for $D<1 \mathrm{~nm}$ For separations as large as $1 \mathrm{~nm}$ the interaction is $O(\mathrm{meV} /$ atom), too small to be found accurately by a full numerical correlation energy calculation using (e.g.) VASP. Thus for this system there is NO range of separations $D$ where we can meaningfully test the full calculation against these asymptotics! Our sub-asymptotic approach in the next Section is more useful in this regard.

\section{B. More useful sub-asymptotic form}

Another way to use (61) and (62) is to leave the screening denominator un-expanded in (61). While we include some $O\left(Q^{2}\right)$ terms by doing this, and so should formally be including all $O\left(Q^{2}\right)$ terms, we have estimated that the denominator in (61) can be far from unity at intermediate layer separations $D$, where the other $O\left(Q^{2}\right)$ terms are still small. With this approach the $Q$ integration can be done analytically (assuming an isotropic layer response in the plane), giving

$$
\begin{aligned}
& \frac{E^{2 \operatorname{Layers}(\text { sub-asy })}(D)}{2 A}=-\frac{1}{2}\left(\frac{1}{2 \pi}\right)^{4} \int_{0}^{\infty} \hbar d u\left[\alpha_{\|}(i u)\right]^{-4} \\
& \times\left(\alpha_{\|}^{2}(i u) f_{2}(\xi)+2 \alpha_{\|}(i u) \alpha_{\perp}(i u) f_{1}(\xi)+\alpha_{\perp}^{2}(i u) f_{0}(\xi)\right)
\end{aligned}
$$


where $\xi \equiv \frac{D}{\pi \alpha_{\|}(i u)}$ and

$$
\begin{aligned}
f_{n}(\xi) & =\int_{0}^{\infty} q^{3} \frac{e^{-|\xi| q}}{(1+q)^{n}} d q \approx 6 \xi^{-4} \text { for } \xi \rightarrow \infty \\
f_{0} & =6 \xi^{-4} \\
f_{1} & =-e^{\xi} E_{1}(\xi)+\xi^{-1}-\xi^{-2}+2 \xi^{-3} \\
f_{2} & =(3+\xi) e^{\xi} E_{1}(\xi)+\xi^{-2}-2 \xi^{-1}-1
\end{aligned}
$$

Here $E_{1}(\xi) \equiv E i(1, \xi)$ is the exponential integral function, item 5.1.1 in Abramowitz and Stegun ${ }^{55}$. Alternatively the $Q$ integration in (39) can be done numerically, which avoids the use of the $E_{1}$ function.

For the infinite stack of uniformly spaced layers (still within a second order expansion of the logarithm) the correlation energy according to (50) is

$$
\frac{E^{\infty \text { stack }(\text { sub-asy) }}(D)}{N A}=2 \sum_{K=1}^{\infty} \frac{E^{2 \operatorname{Layer}(\text { sub-asy })}(K D)}{2 A}
$$

The sum over layer separation index $K$ converges rapidly, like $\sum_{K} K^{-4}$.

In the above arguments, analytic evaluation of the $Q$ integrations depended on perturbative expansion of the logarithm in the full RPA energy expressions, a procedure that we showed to be'valid asymptotically for insulating 2D layers, essentially because 2D intra-layer RPA screening is ineffective as $Q \rightarrow 0$ (This contrasts with thick layers, where conventional Lifshitz theory applies, 3D screening remains signifant as $Q \rightarrow 0$, and the second order expansion of the logarithm is known to make errors of up to $20 \%$ in the vdW energy ${ }^{41}$ ). However one can easily revert to non-expanded logarithmic expressions such as (49) or (38). Numerically exact evaluation for symmetric layers is easily done as a two-dimensional numerical integral over $Q \equiv|\vec{Q}|$ and $u$ for 2 layers, or a $3 \mathrm{D}$ integration on $Q, q_{z}$ and $u$ for the case of an infinite stack. This obtains the correlation energy in seconds to minutes on a laptop machine, in contrast to full RPA energy calculations with a packaged plane-wave code, which takes hours, days or even weeks on a large parallel cluster, even for simple geometries.

\section{INCLUDING $O\left(Q^{2}\right)$ CORRECTIONS IN THE LAYER RESPONSE}

As shown above, the polarizability of a symmetric insulating layer through $O(Q)$ can be determined directly from the macroscopic dielectric function $\varepsilon^{\text {macro }}(Q=0, \omega+i 0)$ of a slightly stretched stack of layers, without further input. However to predict layer interactions at shorter distances one needs the layer response to $O\left(Q^{2}\right)$. At this order many effects come in: (i) local-field $(\vec{G} \neq \overrightarrow{0})$ effects within layers (see $(54,59,60)$ ); (ii) local-field effects between layers; (iii) effects of finite layer width (consider expansion of the exponential in (12) for a symmetric layer); (iv) finite spatial extent of any adsorbates; (v) electron pressure (diffusion) effects in the layer response (for example, the long-wavelenth bare density response of a metallic layer is of form $\left(\chi_{0}(Q, i u)\right)^{-1} \propto u^{2}+\gamma^{2} Q^{2}$ where the diffusion term involves a mean-square velocity spread $\gamma^{2}$ ). It would be too difficult to account for all these effects analytically (or even numerically without complexity comparable to a full RPA correlation energy calculation), so we now introduce an empirical $O\left(Q^{2}\right)$ correction both to the layer response $\alpha^{2 D, s c r}$ and (treated elsewhere) to the polarizability $A(i u)$ of any adsorbate where applicable. Accordingly we write for the layer response through $O\left(Q^{2}\right)$

$$
\begin{aligned}
& \alpha_{x x}^{2 D, s c r}(Q, i u)=\frac{\alpha_{x x}^{2 D, s c r}(Q=0, i u)}{1+2 \pi Q \alpha_{x x}^{2 D, s c r}(Q=0, i u)} \sqrt{1+B^{2} Q^{2}} \exp \left(-(\beta Q)^{6} / 2\right) \\
& \alpha_{z z}^{2 D, s c r}(Q, i u)=\alpha_{x x}^{2 D, s c r}(Q=0, i u) \sqrt{1+B^{2} Q^{2}} \exp \left(-(\beta Q)^{6} / 2\right)
\end{aligned}
$$

where $B$ has dimensions of length and can be regarded as a sort of effective layer width governing polarization properties, and the final exponential factor is discussed below. The square root form in $(69,70)$ is not unique but it allows for the required $O\left(Q^{2}\right)$ correction for small $Q$, while avoiding over-emphasis of large $Q$ values where our theory is at any rate not accurate. Moment expansion of the exponential in (12) suggests that $B$ is real, i.e. that the correction is positive, and this is borne out by the fits to the RPA correlation energy that we obtain below. There really should be separate values $B_{x x}$ and $B_{z z}$ to fit the parallel and perpendicular responses, but here we assume for simplicity that $B_{x x}=B_{z z}=B$. The constant $B$ for each species of layer was fixed by fitting one point on the curve of RPA correlation energy vs layer spacing $D$ of an infinite stack of that particular layer, the fit being done at a point 
that is near to the equilibrium spacing $D_{0}$. Our theory is then used for all other $D$ values, and we shall see that this reproduces the entire interlayer correlation energy curve $E_{c}(D)$ of a stack of layers very well, including $D$ values near to and far from the equilibrium layer spacing $D_{0}$ of the solid. In doing this, we needed to consider one more point, as follows.

At moderate inter-layer distances $D$ the inverse exponential $e^{-2 Q D}$, from the Coulomb potential in correlation energy expressions such as (40), creates a natural cutoff ensuring that the unphysical large- $Q$ behaviour of our small$Q$ resonse expressions $(69,70)$ does not contribute significantly to the energy. However, as the two layers approach their natural binding distance $D_{0}$ this exponential decay is insufficient for the task, and we need to recognize that the $\vec{Q}$ integrations should at least be restricted to the first Brillouin zone. To this end we include an additional damping factor in $(69,70)$ of form $e^{-(\beta Q)^{6}}$. The value of $\beta$ is chosen to satisfy a normalisation condition based on the area $A_{B Z}$ of the 2D Brillouin zone (BZ) of the layer's crystal structure:

$$
\int \exp \left(-(\beta Q)^{6}\right) d^{2} Q=A_{B Z}
$$

This ensures that the energy integration only samples values of $\vec{Q}$ lying roughly within the BZ, even though we formally include larger $Q$ values in our $Q$ integration. This smooth damping factor avoids the numerical difficulties posed by a sharp BZ cutoff in $Q$, but contributes only to $O\left(Q^{6}\right)$ for $Q \rightarrow 0$ and thus does not spoil the desirable small-wavenumber physics of the polarizability model $(69,70)$ that we have created. Apart from this consideration, the use of the 6 th power is not unique.

\section{NUMERICAL RESULTS FOR BN LAYERS}

The results of our procedure for a stretched h-BN crystal are shown in Fig 4. The fully asymptotic energy prediction (51) substantially over-estimates the RPA correlation energy at smaller separations, and so cannot be corrected by a positive $O\left(Q^{2}\right)$ term of the form motivated above on grounds of layer width and other physics. The sub-asymptotic $O(Q)$ form of layer polarizability $(64),(68)$ somewhat under-estimates $E_{c}$ and so is eligible for physically meaningful $O\left(Q^{2}\right)$ correction. By fitting the RPA layer correlation energy at a single point near the equilbrium spacing $D=D_{0}$ we found optimal values $B=0.238 \mathrm{~nm}$ for monolayer $\mathrm{BN}$ and $B=0.265 \mathrm{~nm}$ for monolayer graphene (see (69) and (70)). These choices led to excellent fits to the available RPA interlayer correlation energy at all layer separations $D$ down to $D=D_{0}$, as seen in Fig. 4 . In Fig 5 we also show the same quantity with the perpendicular polarizability $a_{z z}^{2 D}, s c r$ set to zero. This demonstrates that neglect of either one of $a_{z z}^{2 D, s c r}$ or $a_{x x}^{2 D}$,scr yields large errors in the inter-layer correlation energy.

Since our prediction of the interlayer RPA correlation energy alone is very good, in order to reproduce the total RPA energy $E(D)$ we should add the exact Hartree and exchange energies. This gives a very good account of the total RPA binding energy curve $E_{t o t}(D)$ as shown in Fig.6. The convergence parameters of the full RPA energy calculation were as follows: a $12 \times 12 \times 6 \mathrm{k}$-space grid for the exchange calculation, and an $8 \times 8 \times 3$ grid for the correlation energy calculation, reducing to $8 \times 8 \times 1$ for large inter-layer separations. The cut-off for the polarisability matrices was $300 \mathrm{eV}$ and the cut-off of the wavefunction was $700 \mathrm{eV}$.The energies agree well with previous RPA calculations on h-BN ${ }^{31}, 38$

\section{THE SPECIAL CASE OF GRAPHENE}

Graphene is not an insulator and its polarizability is singular as $Q, u \rightarrow 0,0$. Graphene is also a special case because it is very difficult to include enough $\vec{k}$ points, in standard numerical plane-wave codes, to capture the delicate physics of gapless electronic transitions near to the Dirac point, responsible for the graphene's unusual electronic and van der Waals properties. In calculating the layer polarizability for graphene from the plane wave code VASP 49,50 we therefore excised from the numerics any electronic transitions with an energy less than $\varepsilon_{c}=1.25 \mathrm{eV}$, leading to a $2 \mathrm{D}$ polarizability $\alpha^{(i n s)}$ that is insulator-like - i.e. nonsingular as $u \rightarrow 0$. The remaining gapless $\pi_{z}$ transitions give rise to the bare graphenic response from a truncated conical Bloch band ${ }^{33}$. This response is singular as $u \rightarrow 0$ but is known analytically 56,57 . Adding the numerical and analytic contributions we obtain the "rapid" polarizabilities $\alpha^{r a p}$ corresponding to $\chi^{r a p}$ as defined in (55). We note that the $\pi_{z}$ response contains no local field terms and does not contribute to the perpendicular polarizability $\alpha_{z z}$

$$
\alpha_{x x}^{2 D, r a p, g r}(Q, i u)=\alpha_{x x}^{2 D, r a p, i n s, 2 D}(Q, i u)+\alpha^{c o n e}(Q, i u)+O\left(Q^{2}\right)
$$




$$
\alpha^{\text {cone }}=e^{-2}(4 \hbar)^{-1}\left(u^{2}+v_{0}^{2} Q^{2}\right)^{-1 / 2} \theta
$$

Here $v_{0}$ is the characteristic velocity of the graphene electronic bandstructure, and

$$
\theta=\frac{2}{\pi} \arctan \left(\tilde{\varepsilon} / \sqrt{u^{2}+v_{0}^{2} Q^{2}}\right), \quad \tilde{\varepsilon}=\sqrt{\max \left(\varepsilon_{c}^{2}-v_{0}^{2} Q, 0\right)} \rightarrow \varepsilon_{c} \text { as } Q \rightarrow 0 .
$$

Then using (56) to calculate the interacting $\chi^{2 D, s c r}$ we obtain the layer polarizability at small but finite surface-parallel wavenumber in a form including an $O\left(Q^{2}\right)$ correction as in $(69)$ :

$$
\alpha_{x x}^{2 D, \text { scr,graphene }}(Q, i u)=\frac{\alpha_{\|}^{2 D, \text { rap }, g r}(Q, i u)}{1+2 \pi Q \alpha_{x x}^{2 D r a p, g r}(Q, i u)} \sqrt{1+B Q^{2}} \exp \left(-(\beta Q)^{6} / 2\right)+O\left(Q^{3}\right)
$$

where $\alpha_{x x}^{2 D, r a p, g r}$ is given by $(71)$, while $\alpha_{z z}^{2 D, s c r}(Q, i u)=\alpha_{z z}^{r a p}(Q, i u) \sqrt{1+B Q^{2}} \exp \left(-(\beta Q)^{6} / 2\right)+O\left(Q^{3}\right)$ just as for an insulating layer. The cross-correlation energy for two graphene layers is then found by putting (73) into (38) or (39). For an infinite stack of graphenes we put (73) into (49) or (50).

Figure 7 shows the inter-layer correlation energy $E_{c}(D)$ of stretched graphite as a function of layer separation $D$ in the sub-asymptotic approximation, and in our full semianalytic $O\left(Q^{2}\right)$ theory, as well as $E_{c}(D)$ from a full RPA correlation energy calculation using VASP. Agreement of our analytic $O\left(Q^{2}\right)$ theory with the VASP results is excellent over the whole range of $D$ values covered in the figure.

For relatively large layer separations $D$ not covered in Fig 7 (in practice for $D>10 \mathrm{~nm}$ ), graphene layers are known $^{25},{ }^{56}$ to have an unusual van der Waals energy that falls off more slowly than that of insulating layers, as $D^{-3}$ instead of $D^{-4}$.

This asymptotic result can be obtained as follows. For the case of two zero-temperature undoped graphene layers at sufficiently large distance $D$ so that only very small $Q$ values are sampled, the electronic $\pi_{z}$ response dominates, so $\alpha_{1 t o t}=\alpha_{2 t o t}=e^{-2} Q^{-2} \chi, \chi=\chi_{0} /\left(1-2 \pi e^{2} Q^{-1} \chi_{0}\right), \chi_{0}=\left(-Q^{2} / 4 \hbar\right)\left(u^{2}+v_{F}^{2} Q^{2}\right)^{-1 / 2}$. Introducing new dimensionless integration variables $\kappa=Q D, U=u /\left(v_{F} Q\right)$ we reduce (38) to a $D^{-3}$ power law, which has been known for some time ${ }^{25}$ :

$$
\left.\frac{E^{\text {interlayer }}}{A}(2 \text { graphenes })\right)=-\frac{\hbar v_{F}}{(2 \pi)^{2}} D^{-3} \int_{0}^{\infty} \kappa^{2} d \kappa \int_{0}^{\infty} d U \ln \left(1-e^{-2 \kappa}\left(\mu \sqrt{U^{2}+1}+1\right)^{-2}\right)
$$

where $\mu=2 \hbar v_{F} /\left(\pi e^{2}\right)$. Note however that for zero-temperature, undoped graphene layers, in practice this formula is accurate only for layer separations $D$ exceeding about $10 \mathrm{~nm}$ : for smaller separations the insulator-like contributions to the layer response, from non- $\pi_{z}$ electronic transitions, plus finite-Dirac-cone effects, must be included as we have done here, and the $D^{-3}$ dependence is masked by larger terms. The infinite-stack case can be obtained from the layer-pair result as in Eq (50): there is evidence that expanding the logarithm in the RPA correlation energy formula is adequate here (see Table III of ${ }^{58}$ ).

The insert to Fig 7 plots the layer binding energy $E$ of stretched graphite vs. $D^{-3}$ using our Layer Response Theory, for large separations $D>10$ Angstrom where the full RPA energy cannot be obtained meaningfully because of numerical noise. The approximate linear dependence of $E$ on $D^{-3}$ (see (74)) is apparent for large $D$ (left-hand part of the insert). So our LRT has treated all regimes of separation correctly.

For even larger separations the RPA itself is suspect for graphene, and an approximate many-body treatment ${ }^{37}$ suggests that (74) is further modified by a logarithmic factor or even a change to the power of $D$. This is not relevant to the energetics at the $D$ values investigated nuimerically here, however.

Figure 8 shows the total interlayer energy $E_{c}(D)$ of bulk graphite. We include the full RPA correlation energy from a large VASP calculation, and our approximation using (50) and (73) together with the numerically exact exchange energy from VASP. The convergence parameters for the full RPA energy calculation for graphite were as for Ref 33, resulting in an estimated $1 \mathrm{meV}$ /atom numerical uncertainty. Our LRT energies agree with full RPA to about this level. Thus once more the agreement of our extremely efficient analytic $O\left(Q^{2}\right)$ theory with the large costly VASP calculation is excellent.

\section{A. Example: Dispersion interaction in an infinite stack of alternating graphene and BN layers}

For the infinite stack-BN-Gr-BN-Gr-BN-Gr- we use the sum of layer-pair interactions as in (50). Partial justification of use of this log-expanded approximation for graphene layers comes from ${ }^{58}$. The inter-layer correlation energy of a 
graphene-BN pair is obtained from (39) with the layer polarizabilities $\alpha_{1}$ and $\alpha_{2}$ for BN and graphene taken from the working above, with effective layer-width parameters $B^{B N}$ and $B^{g r}$ already determined by the previous fits to pure $\mathrm{BN}$ and pure graphite. The Brillouin zone cutoff parameter $\beta$ was taken as the avarage of those of BN and graphene, since the $2 \mathrm{D}$ lattice parameters are so similar. We also chose the effective layer width parameter $B$ to be the average of that for graphene and that for $\mathrm{BN}$ :

$$
\begin{aligned}
\alpha_{1 t o t} & =\left(\frac{\alpha_{x x}^{B N}(i u)}{1+2 \pi Q \alpha_{x x}^{B N}(i u)}+\alpha_{z z}^{B N}(i u)\right) f \\
\alpha_{2 t o t} & =\left(\frac{\alpha_{x x}^{g r, c u t}(i u)+\alpha^{c o n e}(Q, i u)}{1+2 \pi Q\left(\alpha_{x x}^{g r, c u t}(i u)+\alpha^{c o n e}(Q, i u)\right)}+\alpha_{z z}^{g r, c u t}(i u)\right) f
\end{aligned}
$$

where

$$
f=\sqrt{1+\left(B_{g r}+B_{B N}\right)^{2} Q^{2} / 4} \exp \left(-(\beta Q)^{6} / 2\right)
$$

In Fig. 9 our interlayer correlation energy predictions are compared with those from a full VASP calculation for the hetero-stack. Our semi-analytic $O\left(Q^{2}\right)$ theory gives an excellent fit to the VASP data, without the use of any new fitting parameters specific to this particular hetero-structure. For the full RPA energy calculations on the hetero-stack the convergence parameters were similar to those quoted above for the pure BN calculations.

In Fig 10 we show the total interlayer energy for the BN-gr stack using our Layer Response Theory plus the exact exchange contribution from VASP. Again our results are in excellent agreement with the total interlayer energy from VASP.

\section{SUMMARY}

Our aim here was to provide the necessary semi-analytic correlation energy theory for prediction of RPA-level cohesive energetics on nano-thin layer systems without very large computations. We began by obtaining the longwavelength imaginary-frequency screened polarizablities $\alpha_{x x}^{2 D}, s c r(Q \rightarrow 0, \omega=i u), \alpha_{z z}^{2 D, s c r}(Q \rightarrow 0, \omega=i u)$ of each isolated monolayer species. We did this using efficient calculations of the macroscopic dielectric function $\varepsilon^{\text {macro }}(\omega+i 0)$ for an infinite stack of the relevant layer, with a modestly stretched interlayer spacing $D \approx 2 D_{0}$. This was possible without the use of highly stretched layer spacings because we treated the long-wavelength inter-layer Coulomb screening analytically. See Eqs (30) and (31).

We then used microscopic analytic theory to obtain the finite-wavenumber $O(Q)$ corrections to $\alpha_{i i}^{2 D, s c r}(Q, i u)$ in the presence of all intra-layer local-field $(\vec{G} \neq \overrightarrow{0})$ effects, without further numerical input. See eqs $(61)$ and $(62)$.

For each type of monolayer, we then obtained further $O\left(Q^{2}\right)$ corrections (see Eqs $(69)$ and $(70)$ ) to $\alpha_{i i}^{2 D, s c r}$ by fitting to a single point $D=D_{00} \approx D_{0}$ on the curve of the interlayer correlation energy $E_{c}(D)$ of an infinite stack of the same layer species, from RPA energy calculations using VASP. These large numerical RPA energy calculations were tractable because only a small unit cell is needed to represent such a homogeneous periodic stack. They need to be performed only once for each monolayer species, and are then used for all subsequent calculations involving that species with multiple layer types and other species. This spatially nonlocal physics represented in the $O\left(Q^{2}\right)$ correction puts the theory well outside the normal scope of the traditional Lifshitz approach to dispersion forces, and in particular we obtained good energetics right down to equilibrium binding geometry.

We then used our layer polarizabiltes through $O\left(Q^{2}\right)$ to evaluate the inter-layer correlation energies of layered systems (including the dispersion energy) within the RPA. This could be done semi-analytically because of the simplified $O\left(Q^{2}\right)$ form of the response functions. See (34), (37), (38), (40), (48) and (50).

We found that for layer systems of the types considered, the "standard" single-power $D^{-p}$ asymptotic forms of interaction energy (see (40), (74)) are reasonably correct only for $D>10 \mathrm{~nm}$ and are certainly not valid at inter-layer separations $D$ less than $1 \mathrm{~nm}$ where the correlation energy per atom is of $O(1 \mathrm{meV})$ or more. That is, they are invalid in the regime where codes such as VASP can give meaningful correlation energy results, given practical numerical error considerations. For this regime of relatively small separations, it was necessary to consider the response of each layer through second order in the wavenumber component $Q$ parallel to the layer. This reflects the weak Coulomb screening within a 2D layer as $Q \rightarrow 0$, and the consequent strong $Q$ dependence (spatially non-local character) of the screened layer response. This is in contrast to thick layers, where dielectric prpperties are spatially local in the $Q=0$ limit, and where Lifshitz theory is valid down to moderate non-contacting separations.

We gave the name "Layer Response Theory" (LRT) to this approach that obtains semi-analytically the correlation component of RPA-level interactions involving monolayers, by determining the polarizabilies $a_{i i}^{2 D, s c r}(Q, i u)$ of each 
monolayer species through $O\left(Q^{2}\right)$. As first examples of our LRT method, here we calculated the following quantities semi-analytically, finding excellent agreement with much larger full RPA correlation calculations.

(i) The interlayer correlation energy curve $E_{c}(D)$ for stretched bulk BN, obtaining excellent agreement at all $D$ values with large numerical RPA calculations (see eqs (50),(39), (69), (70) and Fig 4);

(ii) As in (i) but with neglect of the layer-perpendicular polarizability component $\alpha_{z z}^{2 D, s c r}$ (see Fig . 5). This figure shows that both the parallel and perpendicular polarizabilities of BN layers contribute significantly to the interlayer correlation energy. We found a similar situation for graphene (not shown here).

(iii) The total interlayer energy $E_{t o t}(D)$ for stretched bulk BN, using the exact exchange energy from a large VASP calculation. This shows that our semi-analytic correlation energy is good enough for quantitative predictions even near to the equilibrium spacing. (See Fig 6)

(iv) The interlayer correlation energy of an infinite stack as in (i), but for graphite instead of BN. See Eqs (50) and (73) and Fig 7. Agreement with large numerical RPA calculations is excellent.

(v) The total interlayer energy of stretched graphite using our Layer Response Theory for correlation, plus exact exchange from VASP: see Fig 8. Agreement with large VASP total RPA energy calculations is excellent.

(vi) The correlation and total interlayer energies for an infinite BN-gr-BN-gr-BN-gr...hetero-stack (Eqs (75), (76),(50), (39) and Figs 9, 10). Once more agreement with a large VASP RPA energy calculation was excellent, and here we did not use any external input data for this specific heterostructure. All data (one parameter each) came from VASP calculations of the pure graphene and pure BN stacks.

The energies of physisorbed molecular species on graphene constitute another application that we will discuss elsewhere. We will report successful semi-analytic calculations for this case also.

Our experience so far suggests that the correlation energies are remarkably smooth functions of layer separation $D$ and not strongly dependent on layer-layer registry. A similar conclusion was reached by Loncaric and Despoja ${ }^{13}$ where a metal surface near to a graphene sheet was successfully treated in the jellium model. This smoothness is less true for the Hartree and exchange contributions, which contain most of the dependence on the registration of atoms on adjacent layers. Semilocal energy functionals can give a reasonable account of such registry energies near to contact separations. With some empiricism, it is possible to meld our accurate semi-analytic correlation energies with results from semi-local theories of exchange and correlation. See for example ${ }^{59}$. We will pursue this elsewhere.

Another promising direction is the inclusion of beyond-RPA effects in the correlation energy via simplified exchangecorrelation kernels. See for example ${ }^{60},{ }^{36}, 61$.

\section{ACKNOWLEDGMENTS}

We thank Prof. A. Rubio, Dr. T. Bjorkman, Prof. K. Thygesen, Prof. V. Despoja and Prof E. M. Gray for discussions. The authors benefited from Griffith University funding in the form of a Short Term Visiting Research Fellowship (2015). Earlier financial support came from Australian-French government funding under the FAST scheme. We also acknowledge financial support from CNRS (Centre National de la Recherche Scientifique) through the PICS program "2DvdW". JFD acknowledges support from CNRS and University of Lorraine during visits to Nancy.

${ }^{1}$ K. S. Novoselov, A. K. Geim, S. V. Morozov, D. Jiang, Y. Zhang, S. V. Dubonos, I. V. Grigorieva, and A. A. Firsov, Science 306, 666 (2004).

${ }^{2}$ K. S. Novoselov, D. Jiang, F. Schedin, T. J. Booth, V. V. Khotkevich, S. V. Morozov, and A. K. Geim, Proceedings of the National Academy of Sciences of the United States of America 102, 10451 (2005).

3 J. N. Coleman, M. Lotya, A. ONeill, S. D. Bergin, P. J. King, U. Khan, K. Young, A. Gaucher, S. De, R. J. Smith, et al., Science 331, 568 (2011).

${ }^{4}$ K. F. Mak, C. Lee, J. Hone, J. Shan, and T. F. Heinz, Physical Review Letters 105, 136805 (2010).

5 A. K. Geim and K. S. Novoselov, Nature Materials 6, 183 (2007).

${ }^{6}$ A. H. Castro Neto, F. Guinea, N. M. R. Peres, K. S. Novoselov, and A. K. Geim, Reviews of Modern Physics 81, 109 (2009).

7 M. Xu, T. Liang, M. Shi, and H. Chen, Chemical Reviews 113, 3766 (2013).

8 L. Liao, H. Peng, and Z. Liu, Journal of the American Chemical Society 136, 12194 (2014).

9 A. K. Geim and I. V. Grigorieva, Nature 499, 419 (2013).

10 M. Amft, S. Lebègue, O. Eriksson, and N. V. Skorodumova, J. Phys. Cond. Mat. 23, 395001 (2011).

11 S. Javaid, S. Lebègue, B. Detlefs, F. Ibrahim, F. Djeghloul, M. Bowen, S. Boukari, T. Miyamachi, J. Arabski, D. Spor, et al., Phys. Rev. B 87, 155418 (2013).

12 W. Liu, J. Carrasco, B. Santra, A. Michaelides, M. Scheffler, and A. Tkatchenko, Physical Review B 86, 245405 (2012).

13 I. Loncaric and V. Despoja, Phys. Rev. B 90, 075414 (2014).

14 S. Zhu and T. L, ACS Nano 8, 2864 (2014). 
15 B. V. Cunning, M. Ahmed, N. Mishra1, A. R. Kermany, B. Wood, and F. Iacopi, Nanotechnology 25, 25301 (2014).

16 L. Tao, E. Cinquanta, D. Chiappe, C. Grazianetti, M. Fanciulli, and M. Dubey, Nature Nanotechnology 10, 227 (2015).

17 F. Iacopi, N. Mishra, B. V. Cunning, D. Goding, S. Dimitrijev, R. Brock, R. H. Dauskardt, B. wood, and J. Boeckl, J. Mater. Res 30, 609 (2015).

18 J. Antony, S. Grimme, S. Ehrlich, and H. Krieg, J. Chem. Phys. 132, 154104 (2010).

19 R. Sure, J. Antony, and S. Grimme, J. Phys. Chem. B 118, 3431 (2014).

20 S. Grimme, Journal of Computational Chemistry 27, 1787 (2006).

21 A. Tkatchenko and M. Scheffler, Physical Review Letters 102, 073005 (2009).

22 T. Risthaus and S. Grimme, Journal of Chemical Theory and Computation 9, 1580 (2013).

${ }^{23}$ H. Kim, J.-M. Choi, and W. A. Goddard, The Journal of Physical Chemistry Letters 3, 360 (2012).

24 M. Dion, H. Rydberg, E. Schröder, D. C. Langreth, and B. I. Lundqvist, Phys. Rev. Lett. 92, 246401 (2004).

25 J. F. Dobson, A. White, and A. Rubio, Phys. Rev. Lett. 96, 073201 (2006).

26 A. Tkatchenko, R. A. DiStasio, Jr., R. Car, and M. Scheffler, Phys. Rev. Lett. 108, 236402 (2012).

27 V. Gobre and A. Tkatchenko, Nature Communications 4, 2341 (2013).

28 B. Sernelius, Europhys. Letts. 95, 57003 (2011).

29 A. Ruzsinszky, J. Perdew, J. Tao, G. I. Csonka, and J. M. Pitarke, Phys. Rev. Lett. 109, 233203 (2012).

30 J. F. Dobson and J. Wang, Phys. Rev. Lett. 82, 2123 (1999).

31 A. Marini, P. Garcia-Gonzalez, and A. Rubio, Phys. Rev. Lett. 96, 136404 (2006).

32 J. Harl and G. Kresse, Phys. Rev. Lett. 103, 056401 (2009).

33 S. Lebegue, J. Harl, T. Gould, J. G. Angyan, G. Kresse, and J. F. Dobson, Phys. Rev. Lett. 105, 196401 (2010).

${ }^{34}$ D. Lu, Y. Li, D. Rocca, and G. Galli, Physical Review Letters 102, 206411 (2009).

35 A. Gruneis, M. Marsman, J. Harl, L. Schimka, and G. Kresse, J. Chem. Phys. 131, 154115 (2009).

36 T. Olsen and K. S.Thygesen, Phys. rev. Lett. 112, 203001 (2014).

37 J. F. Dobson, T. Gould, and G. Vignale, Phys. Rev. X 4, 021040 (2014).

38 T. . Bjorkman, A. Gulans, A. V. Krasheninnikov, and R. M. Nieminen, J. Phys.: Condens. matter 24, 424218 (2012).

39 T. Olsen and K. S. Thygesen, Phys. Rev. B 87, 075111 (2013).

40 R.-F. Liu, J. G. Angyan, and J. F. Dobson, J. Chem. Phys 134, 114106 (2011).

41 I. E. Dzyaloshinskii, E. M. Lifshitz, and L. P. Pitaevskii, Adv. Phys. 10, 165 (1961).

42 V. A. Parsegian, van der Waals Forces: a handbook for biologists, chemists, engineers, and physicists (Cambridge University Press, Cambridge, 2006), ISBN 0521839068, 9780521839068.

43 J. Israelachvili, Intermolecular and Surface Forces, (Academic, London, 1992): J. Mahanty and B. Ninham, Dispersion Forces, (Academic, London, 1976): D. Langbein, Theory of van der Waals Attraction (Springer-Verlag, Berlin) (1974).

44 S. J. Rahi, T. Emig, N. Graham, R. L. Jaffe, and M. Kardar, Phys. Rev. D 80, 085021 (2009).

45 S. Scheel and S. Y. Buhmann, Acta Physica Slovaca 58, 675 (2008).

46 S. Y. Buhmann, Dispersion Forces I (Springer-Verlag, Berlin, 2012), ISBN 978-3-642-32483-3, 978-3-642-32484-0.

47 S. Y. Buhmann, Dispersion Forces II (Springer-Verlag, Berlin, 2012), ISBN 978-3-642-32465-9, 978-3-642-32466-6.

48 J. F. Dobson and T. Gould, J. Phys. Condens. Matter 24, 073201 (2012).

49 G. Kresse and D. Joubert, Phys. rev. B 59, 1758 (1999).

${ }^{50}$ G. Kresse and J. Furthmuller, Phys. rev. B 54, 11169 (1996).

51 V. U. Nazarov, New J. Phys. 17, 073018 (2015).

${ }^{52}$ K. Andersen, S. Latini, and K. S. Thygesen, Nanoletters 15, 4616 (2015).

53 J. F. Dobson and B. P. Dinte, Phys. Rev. Lett. 76, 1780 (1996).

54 J. F. Dobson, B. Dinte, and J. Wang, in Electronic Density Functional Theory: Recent Progress and New Directions, edited by J. F. Dobson, G. Vignale, and M. P. Das (Plenum, New York, 1998), chap. 18, p. 261.

55 M. Abramowitz and I. A. Stegun, Handbook of mathematical functions (Dover, Toronto, 1965).

56 T. Gould, J. F. Dobson, and S. Lebegue, Phys. Rev. B 87, 165422 (2013).

57 J. F. Dobson, Surf. Sci. 605, 960 (2011).

58 J. F. Dobson, J. Comp. Theoret. Nanosci. 6, 960 (2009).

59 T. Gould, S. Lebegue, and J. F. Dobson, J. Phys. Condens. Matter 25, 445010 (2013).

60 T. Olsen and K. S.Thygesen, Phys. Rev, B 88, 115131 (2013).

61 T. Gould, J. Chem. Phys 137, 111101 (2012). 


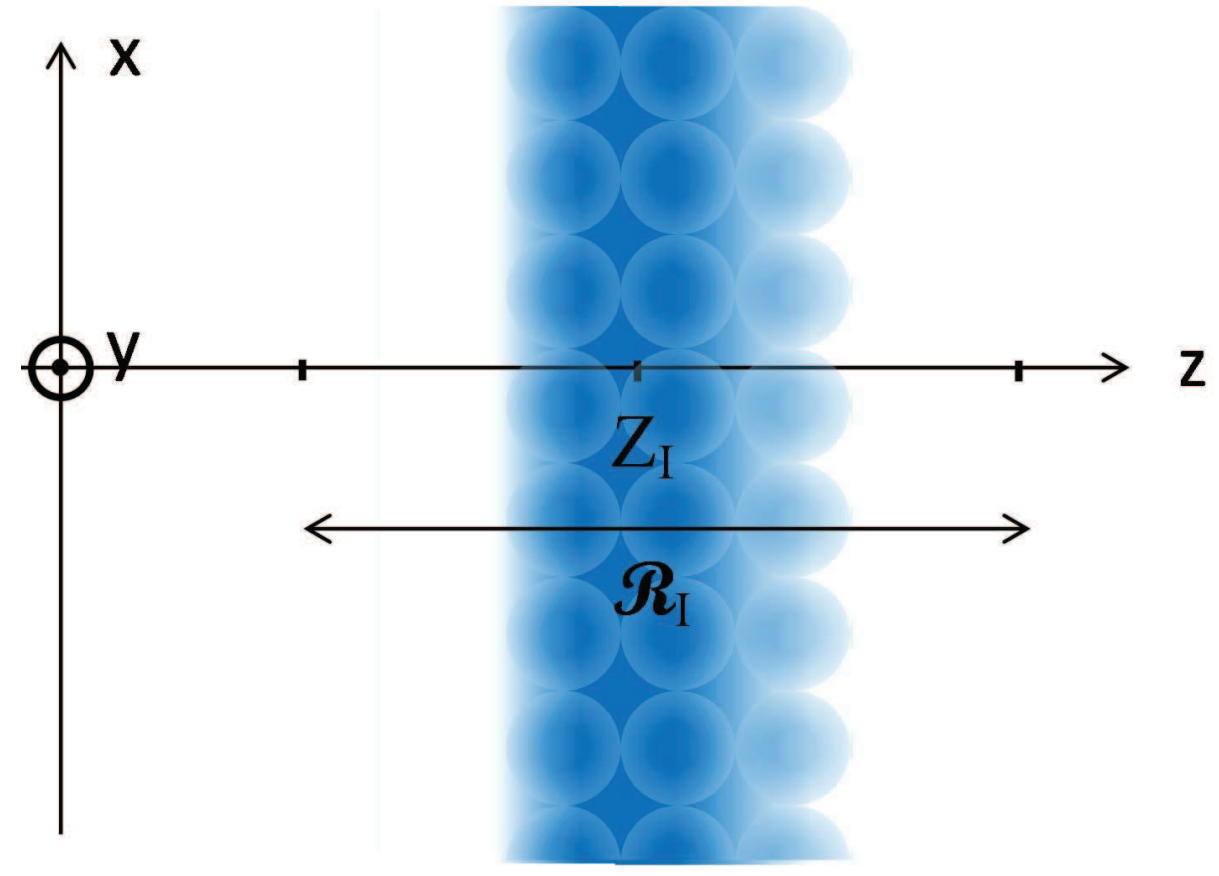

FIG. 1: Scheme for division of the $z$ axis 
a)

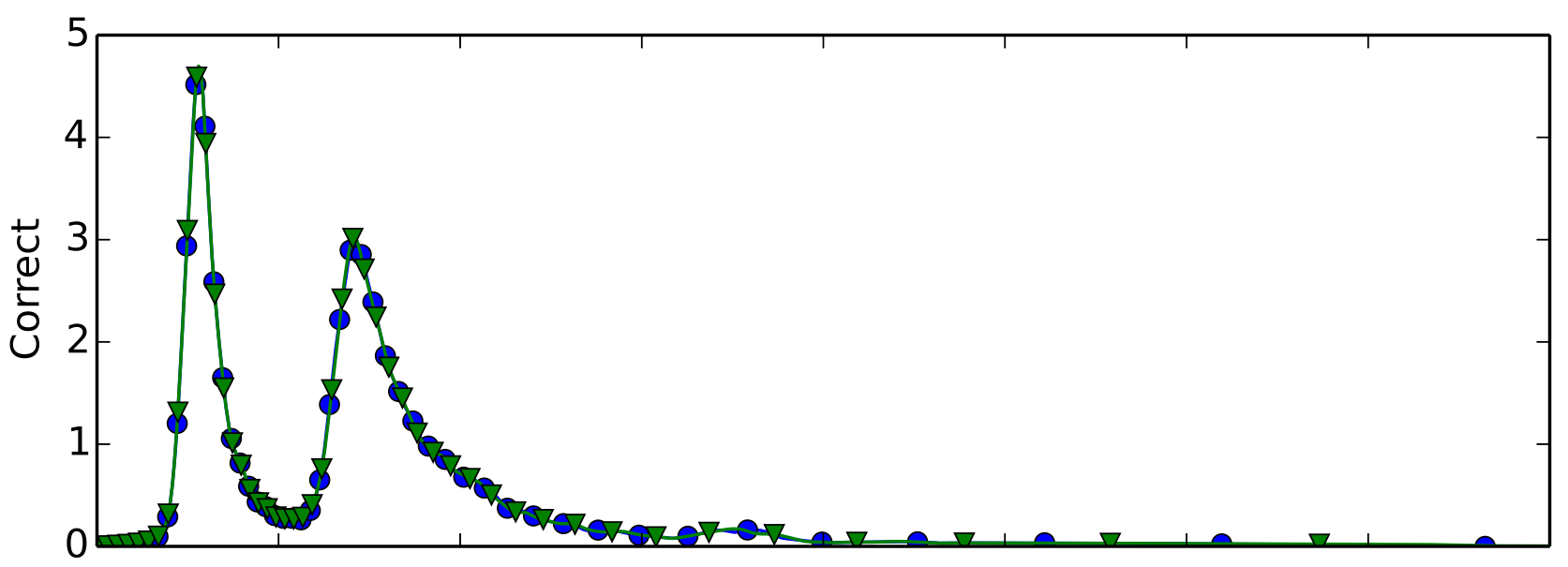

b)

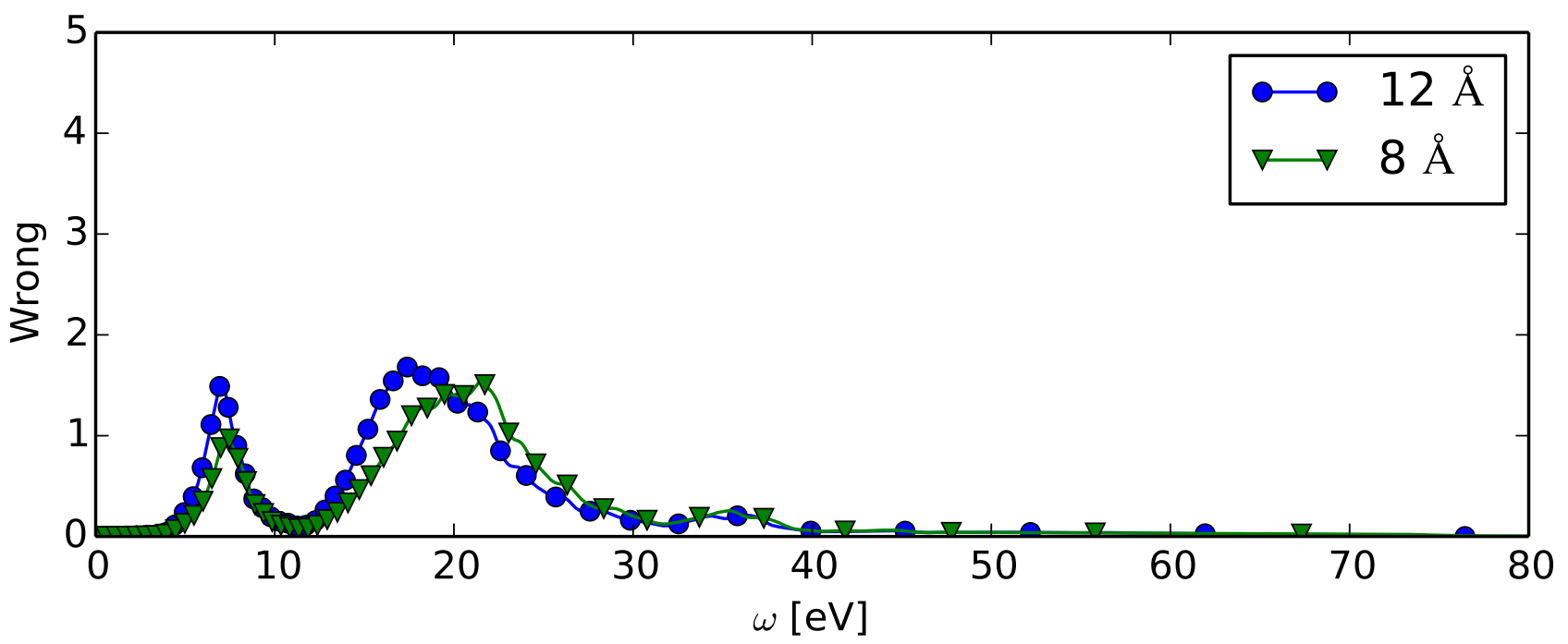

FIG. 2: (a) Parallel-field layer polarizabilites $\operatorname{Im}\left(\alpha_{x x}^{2 D}\right.$, scr $\left.(\omega+i 0)\right)$ deduced, via the correct Eq (30), from VASP $\varepsilon_{x x}^{m a c r o}$ data for solid $\mathrm{BN}$ with stretched lattice spacings $D=0.8 \mathrm{~nm}$ (triangles) and $D=1.2 \mathrm{~nm}$ (circles). The agreement for the two $D$ values is excellent, verifying that (30) correctly allows for the interaction between the layers. (b) As in (a), except that the wrong formula (31) (appropriate to $\epsilon_{z z}$ isntead of $\epsilon_{x x}$, and reflecting lack of inter-layer interaction) was used. Now the cases $D=0.8 \mathrm{~nm}$ and $D=1.2 \mathrm{~nm}$ yield distinctly different results for $\operatorname{Im}\left(\left(\alpha_{x x}^{2 D}, s c r\right)^{2}\right)$, though one could still in principle obtain the correct $\alpha_{x x}^{2 D, s c r}(\omega+i 0)$ by going to the $D \rightarrow \infty$ case, where the layers truly do not interact. 

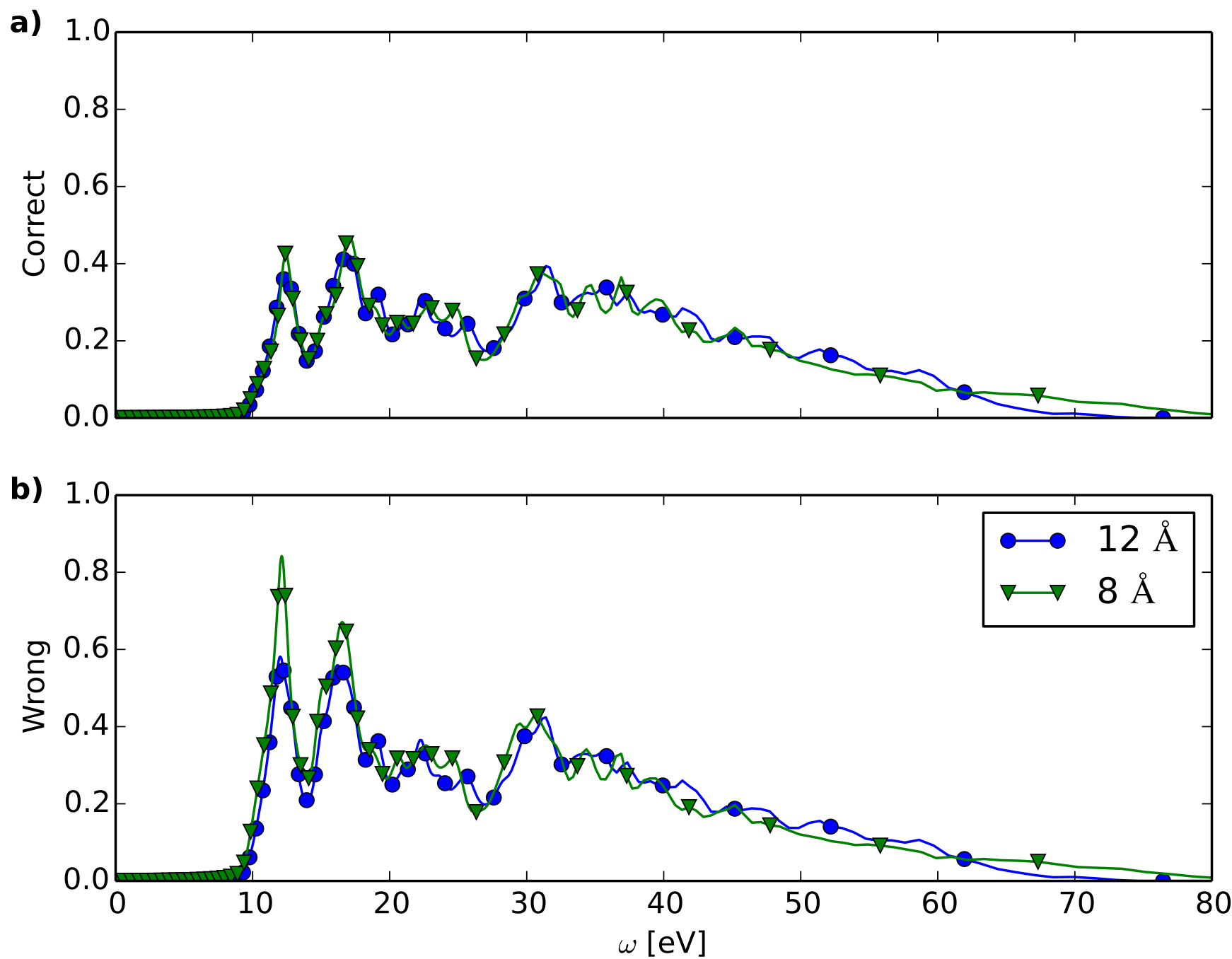

FIG. 3: (a) Field-perpendicular layer polarizabilites $\operatorname{Im}\left(\alpha_{z z}^{2 D, s c r}(\omega+i 0)\right)$ deduced via Eq (31), from VASP $\varepsilon_{z z}^{\text {macro }}$ data for solid $\mathrm{BN}$ with stretched lattice spacings $D=0.8 \mathrm{~nm}$ (triangles) and $D=1.2 \mathrm{~nm}$ (circles). The agreement for the two $D$ values is good.

(b) As in (a) but with unjustified use of the field-parallel formula (30). Agreement between $D=0.8 \mathrm{~nm}$ and $D=1.2 \mathrm{~nm}$ is now not so good. 


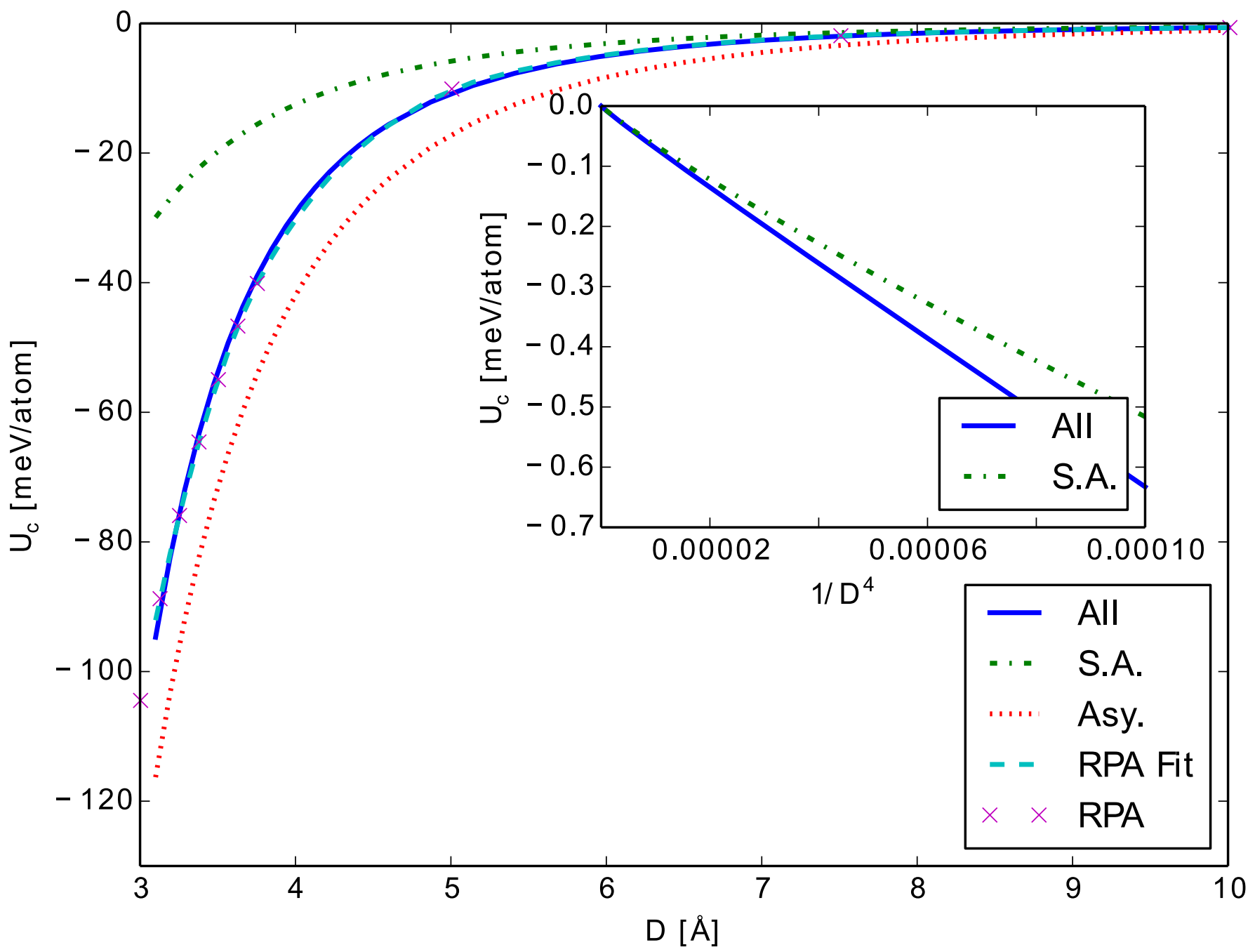

FIG. 4: Inter-layer correlation energy $U_{c} \equiv E_{c}(D)$ per atom $(\mathrm{meV})$ of stretched bulk BN with layer spacing $D$. Short green dashes with pale blue crosses: RPA correlation energy from VASP. Red dots: asymptotic $D^{-4}$ result from Eq (51). Dark green dash-dots: sub-asymptotic energy from Eqs (64) and (50). Solid blue line: our semi-analytic result $(O(Q)$ sub-asymptotics plus $O\left(Q^{2}\right)$ correction) from (39), (50), (69) and (70). Insert: $E_{c}$ vs $D^{-4}$ (Angstrom $^{-4}$ ) for larger $D$ values, showing approximate proportionality $E_{c} \approx K D^{-4}$. 


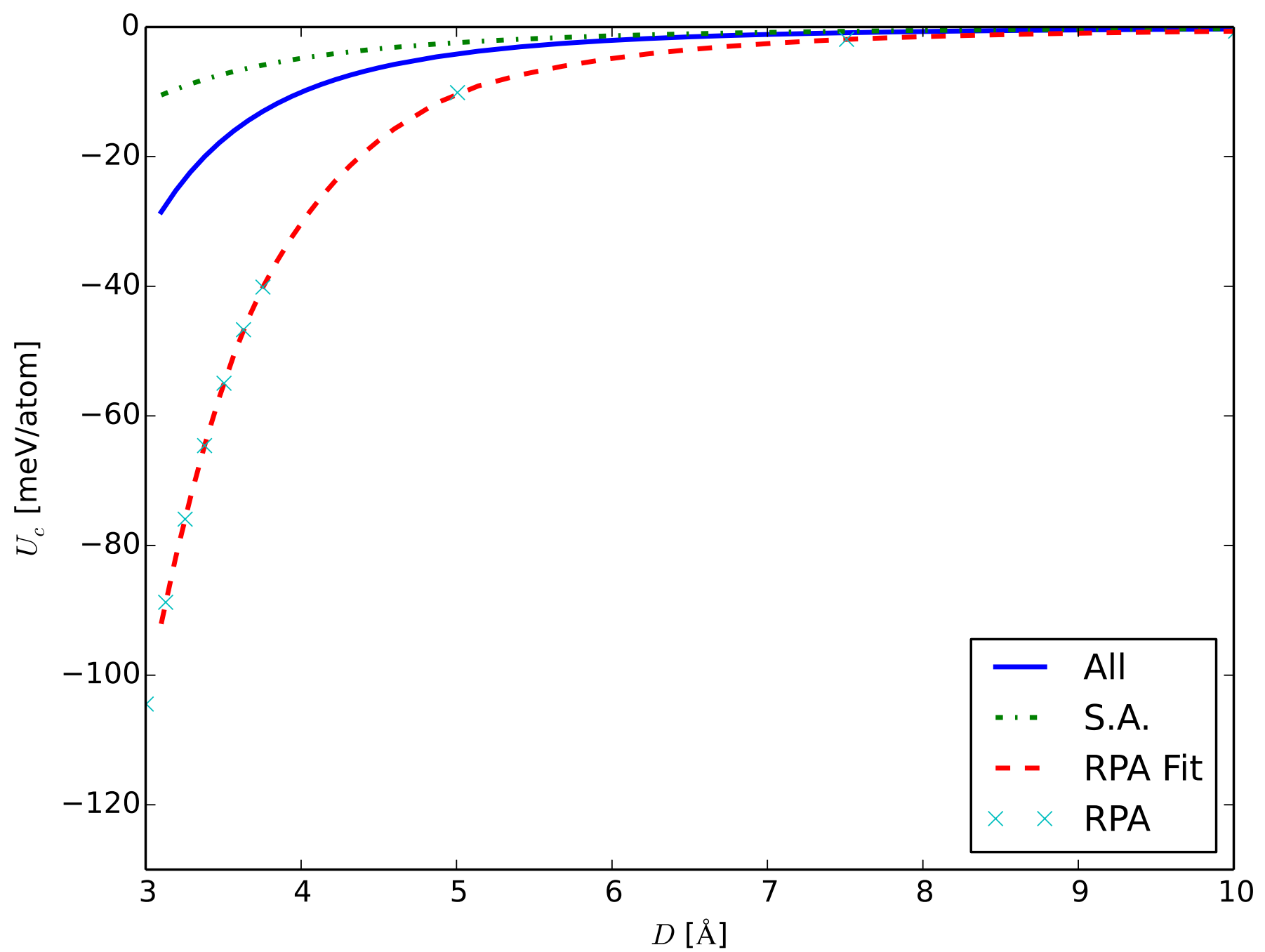

FIG. 5: Interlayer correlation energy $U_{c} \equiv E_{c}(D)$ (meV/atom) of stretched bulk BN using only the parallel response, showing the importance of including both perpendicular and parallel layer polarizability in the analytic work. Red dashed line (with light blue crosses at the numerical data points): Full $E_{c}(D)$ from numerical RPA using VASP. Solid blue line: Our best analytic fit, but with the perpendicular polarizability $\alpha_{z z}^{2 D, s c r}$ set to zero. Green dash-dots: Our sub-asymptotic energy (64) with $\alpha_{z z}^{2 D}, s c r$ set to zero. Note the poor fit of our best theory to full RPA data here when $\alpha_{z z}^{2 D, s c r}$ set to zero, in contrast to the excellent fit in Fig 4 where both $\alpha_{z z}^{2 D, s c r}$ and $\alpha_{x x}^{2 D, s c r}$ are included. 


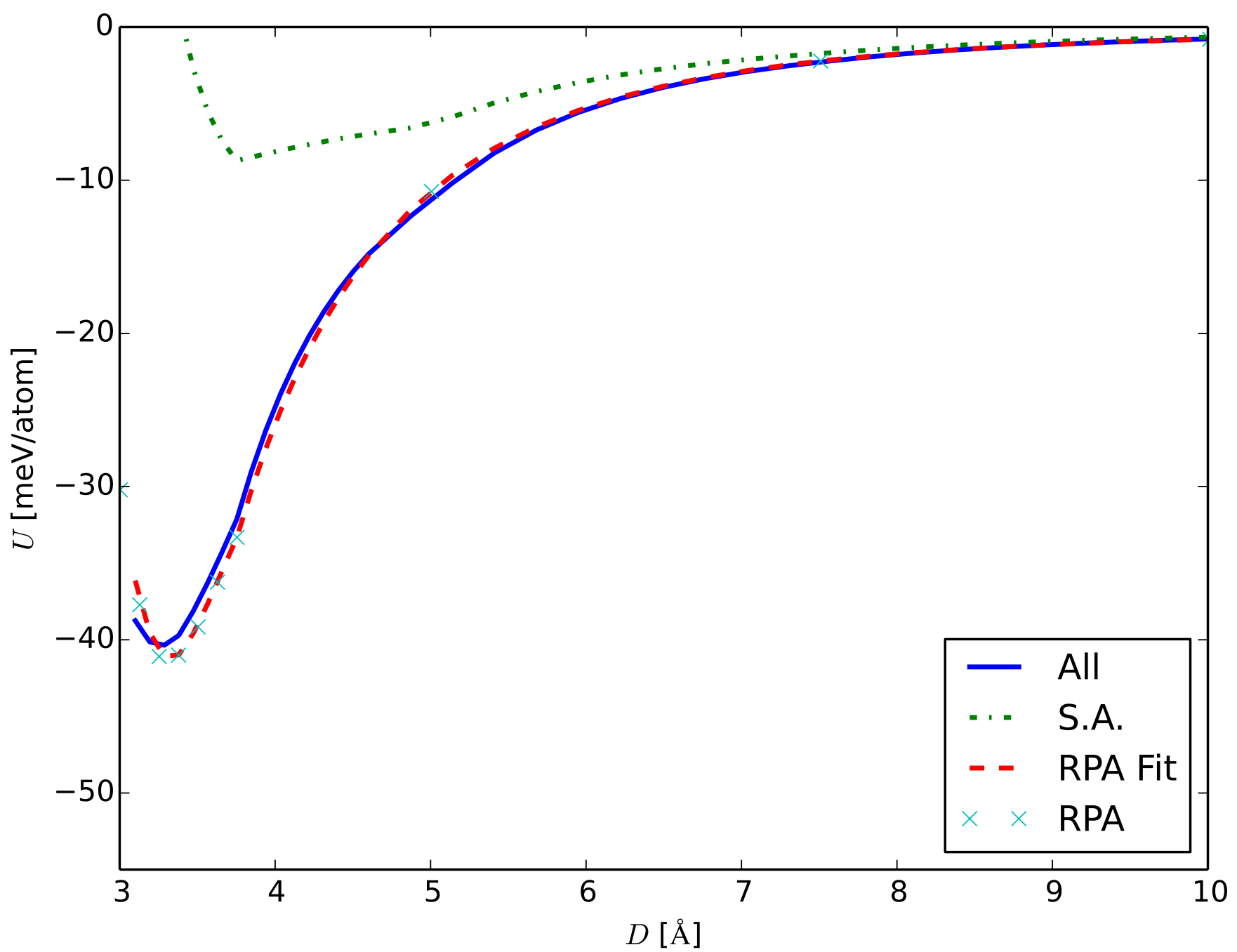

FIG. 6: Total interlayer energy $U \equiv E_{t}$ ot $(D)$ of stretched bulk BN (meV/atom), including exact exchange energy fom VASP in all cases. Red dashes with blue-green crosses: with umerically exact RPA correlation energy from VASP. Solid blue line: with correlation energy from our best semi-analytic theory (see also Fig 4). Dark green dash-dots: with sub-asymptotic correlatiom energy from (64) and (50). (51). 


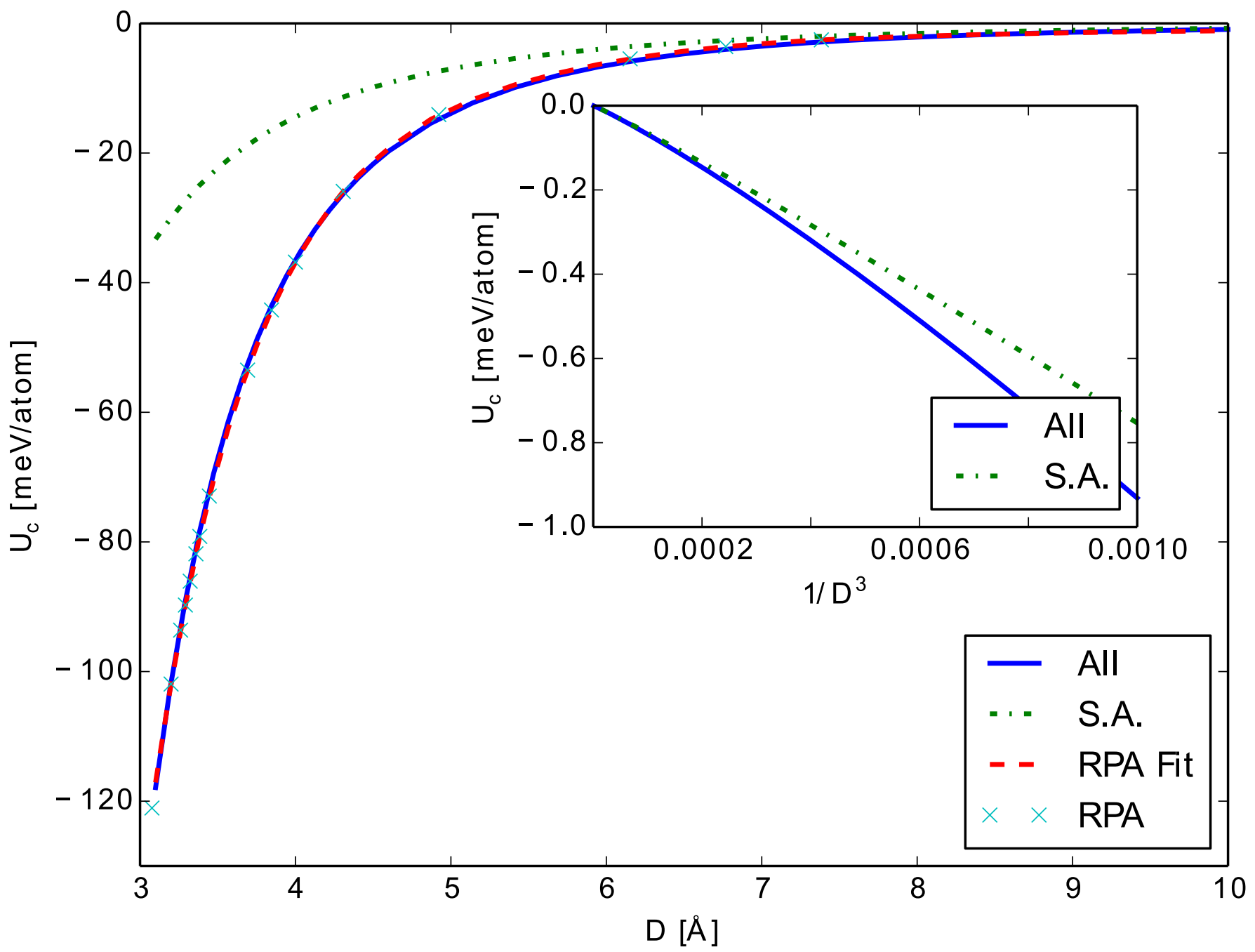

FIG. 7: Interlayer correlation energy $E_{c}(D)$ of stretched graphite (meV/atom). Red dashes: RPA correlation energy from VASP, with individual data points shown by crosses. Blue solid line: our best semi-analytic (sub-asymptotic $+O\left(Q^{2}\right)$ ) theory from (73), (39) and (50). Green dash-dots: our sub-asymptotic theory. . Insert: energy vs $D^{-3}$ for $D>1 \mathrm{~nm}$. See also ref. 56 . 


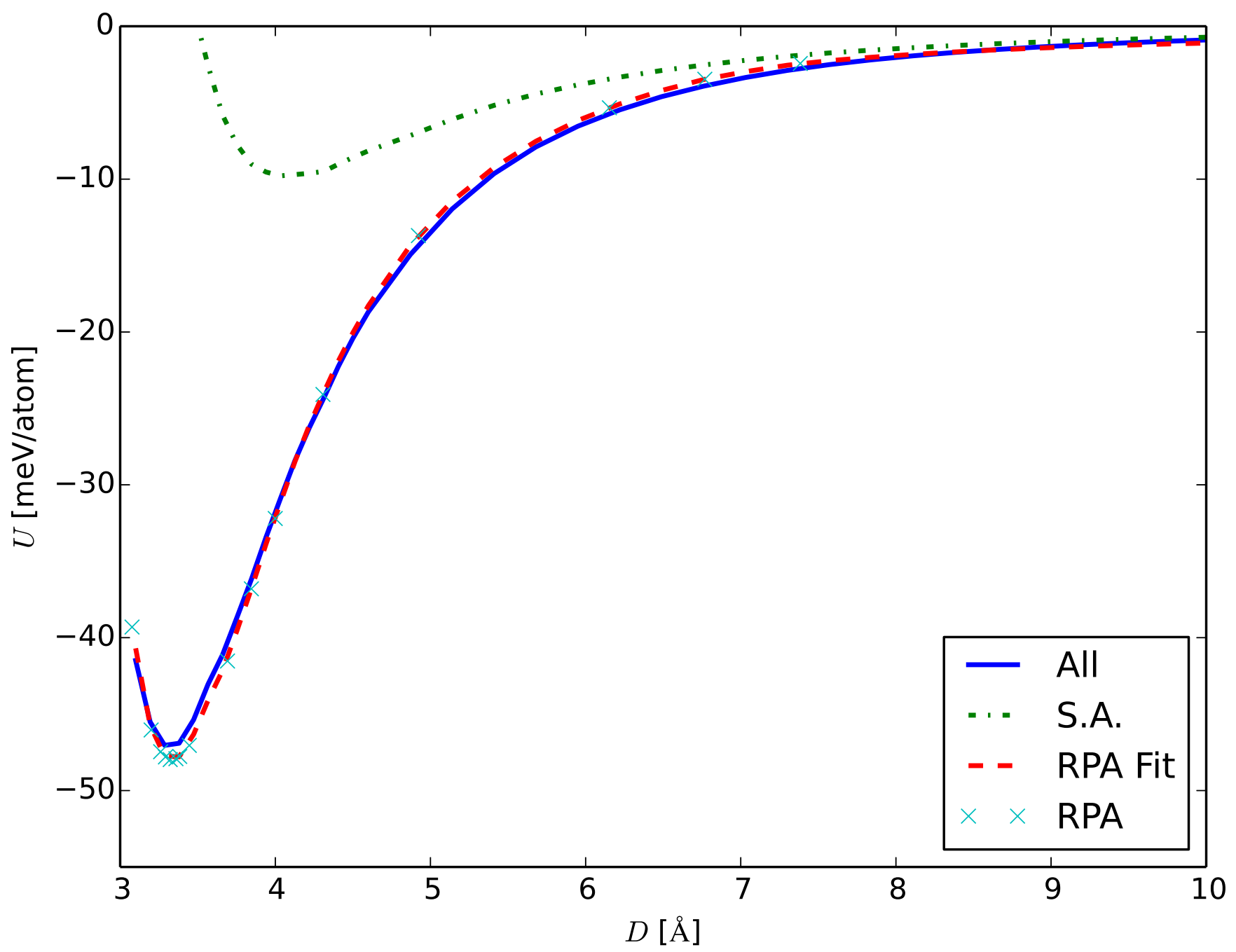

FIG. 8: Total interlayer energy $U \equiv E_{\text {tot }}(D)$ of stretched graphite (meV/atom). Red dashes with crosses: numerically exact RPA energy including exact exchange energy, from VASP. Solid blue line: using correlation energy from our best semi-analytic theory plus Hartree and exact exchange energy from VASP. Green dash-dots: our sub-asymptotic approximation. 


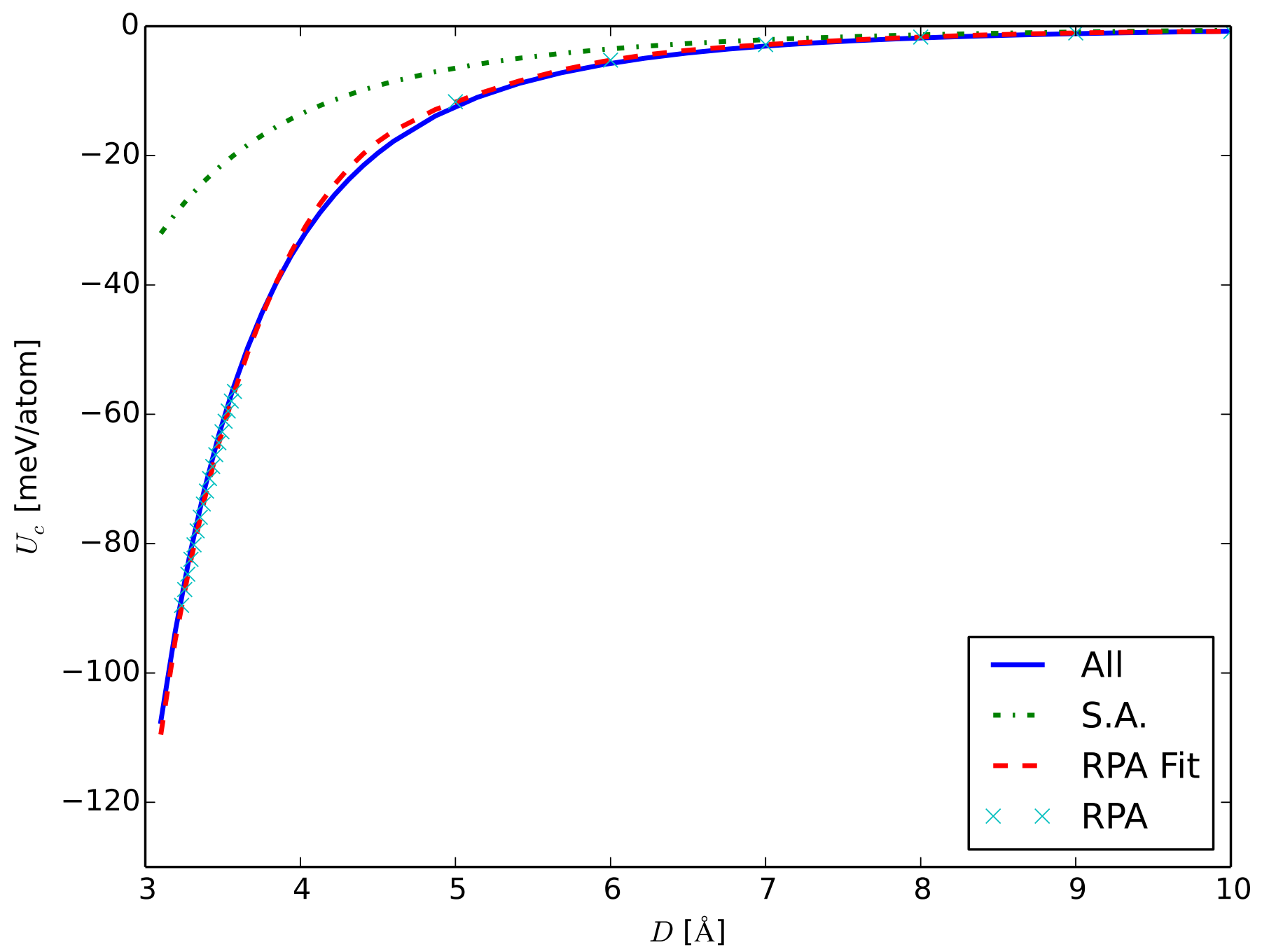

FIG. 9: Interlayer correlation energy $U_{c} \equiv E_{c}(D)$ from an infinite stretched stack ...BN-gr-BN-gr...of alternating graphene and BN layers. Red dashes with crosses: RPA correlation energy from VASP. Green dash-dots: our sub-asymptotic formula. Blue solid line: our best Layer Theory prediction through $O\left(Q^{2}\right)$ from $(50),(39),(61)$ and (73). 


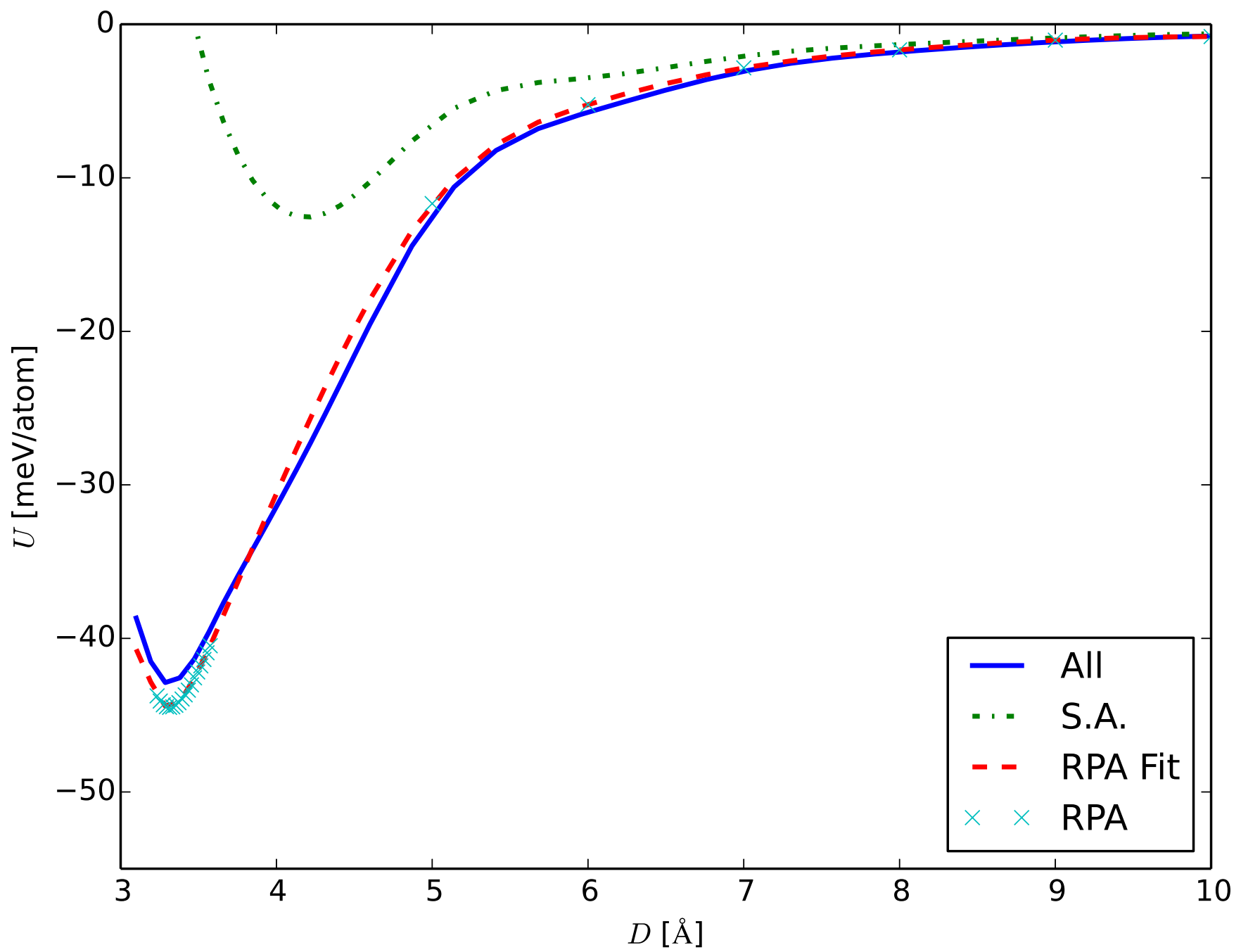

FIG. 10: Total interlayer energy $U \equiv E_{t o t}(D)$ from an infinite stretched stack ...BN-gr-BN-gr...of alternating graphene and BN layers (meV/atom), including exact exchange energy fom VASP. Color scheme of graph as for Fig. 9. 